\title{
Influence on strain-rate history effects on the development of necking instabilities under dynamic loading conditions
}

\author{
N. Jacques ${ }^{\text {a,* }}$, J.A. Rodríguez-Martínez ${ }^{\text {b }}$ \\ ${ }^{a}$ ENSTA Bretagne, UMR CNRS 6027, IRDL, 2 rue François Verny, F-29806 Brest cedex 9, France \\ ${ }^{b}$ Department of Continuum Mechanics and Structural Analysis. University Carlos III of Madrid. \\ Avda. de la Universidad, 30. 28911 Leganés, Madrid, Spain
}

\begin{abstract}
The present paper is devoted to the analysis of strain-rate history effects on neck formation under dynamic loading. For materials presenting strain-rate history effects, two different strain-rate sensitivities should be distinguished: the instantaneous strain-rate sensitivity and the work-hardening strain-rate sensitivity. We have analysed the relative contributions of these two kinds of strain-rate sensitivities to neck retardation for two different configurations: a bar under impact tension and a dynamically expanding ring. For this purpose, we have developed finite element models and, for the second configuration, an analytical model based on the linear stability analysis. The obtained results show that strain-rate history effects have a significant influence on the onset and development of necking. The reason of this phenomenon is that, contrary to the instantaneous strain-rate sensitivity, the work-hardening strain-rate sensitivity does not contribute to delay the neck formation.
\end{abstract}

Keywords: Necking; Dynamic loading; Plastic flow localisation; Critical impact velocity; Viscoplasticity; Strain-rate history effects.

\section{Introduction}

It is well established that the occurrence and development of necking instabilities is strongly related to the work hardening and strain-rate sensitivity of the material. Woodford (1969) analysed results of uniaxial tensile tests for different materials and observed a correlation between the total elongation at failure and the strain-rate sensitivity of the materials. Several

\footnotetext{
* Corresponding author. Tel.: +33 2983489 36; fax: +33 298348730 .

E-mail address: nicolas.jacques@ensta-bretagne.fr (N. Jacques)
} 
theoretical and computational models have been proposed to rationalise the effect of strain-rate sensitivity on necking (Marciniak et al., 1973; Ghosh, 1977a; Hutchinson and Neale, 1977; Chung and Wagoner, 1988; Wagoner and Wang, 1983; Audoly and Hutchinson, 2019). These studies have shown that a rather small amount of strain-rate sensitivity can significantly delay the occurrence of localised necking. In this regard, it should be noted that the predictions of localisation analyses are generally very sensitive to the adopted constitutive relation. For instance, when a simple power-law relation (for which the strain-rate sensitivity is constant) is employed, the necking strain is independent of the prescribed strain-rate (Hutchinson and Neale, 1977). With more advanced constitutive laws, an increase in strain-rate may either increase or decrease the ductility (Xue et al., 2008; Ghosh, 1977b).

At high strain rate, inertia causes an additional stabilising effect on necking (Fressengeas and Molinari, 1985; Altynova et al., 1996). Both mechanisms (inertia and strain-rate sensitivity) contribute to hinder neck development and delay the occurrence of plastic flow localisation (Mercier and Molinari, 2003; Xue et al., 2008). However, the interplay between inertia and strain-rate sensitivity is complex and, in some cases, increasing rate sensitivity may lead to a less stable material behaviour (Vadillo et al., 2012; Jacques, 2020).

In some circumstances, inertia may have a detrimental effect on ductility, mainly because of wave propagation effects. For instance, in linear specimens initially at rest and subjected to dynamic impact tension, the location of the necks is controlled by the intervention of stress waves (Nemes and Eftis, 1993; Vaz-Romero et al., 2015; Rittel et al., 2014; Rotbaum et al., 2015; Needleman, 2018). Moreover, wave propagation effects are responsible for the existence of a critical impact velocity (CIV) which defines the maximum energy absorbed by the sample and the maximum sample ductility in the dynamic tension test (von Karman and Duwez, 1950; Wood, 1967; Klepaczko, 2005; Rusinek et al., 2005; Rodríguez-Martínez et al., 2013b). The CIV corresponds to the lowest impact-velocity which induces sample failure close to the impacted end due to plastic waves trapping. When the velocity applied at the end of the specimen exceeds the CIV, plastic deformation does not spread along the gauge of the specimen, it is "trapped" near the impacted end of the sample. If the applied velocity is further increased, the energy absorbed by the specimen drops rapidly. Note that several numerical studies showed a relation between CIV and material strain-rate sensitivity (increasing strainrate sensitivity leads to a larger CIV) (Hu and Daehn, 1996; Vaz-Romero and RodríguezMartínez, 2019). In contrast, no critical velocity is observed in experiments on dynamic expansion of rings (Niordson, 1965; Altynova et al., 1996; Zhang and Ravi-Chandar, 2006, 
2018) and cylinders (Wood, 1967; Zhang and Ravi-Chandar, 2010; Jeanson et al., 2016). In these experiments, the radial symmetry of the specimen and the loading enables to obtain a uniform strain field (prior to the occurrence of plastic flow instabilities), even at very high loading velocity.

Moreover, it should be mentioned that most of the theoretical and numerical works devoted to the analysis of the influence of strain-rate sensitivity on necking instabilities consider "standard" viscoplastic models in which the flow stress of the material depends on the current values of the effective plastic strain and strain-rate. However, many materials notably Face Centred Cubic (FCC) and Hexagonal Close Packed (HCP) metals exhibit strain-rate history effects (Nicholas, 1971; Klepaczko, 1975; Senseny et al., 1978; Lea, 2018). This means that the flow stress does not only depend on the instantaneous (actual) value of the strain rate, but also on its prior evolution. Fig. 1 shows schematic stress-strain curves illustrating strain-rate history effects (as typically observed in FCC and HCP materials, see for e.g. (Klepaczko, 1975) and (Senseny et al., 1978)). The two solid lines correspond to the responses of the material at constant strain rates $\dot{\varepsilon}_{1}$ and $\dot{\varepsilon}_{2}$ (with $\dot{\varepsilon}_{2}>\dot{\varepsilon}_{1}$ ) and the dotted line to the case when a sudden strain-rate increment from $\dot{\varepsilon}_{1}$ to $\dot{\varepsilon}_{2}$ occurs during the test. Notice that the flow stress after the strain-rate jump is significantly lower than if the material had been deformed at $\dot{\varepsilon}_{2}$ since the beginning of the test.

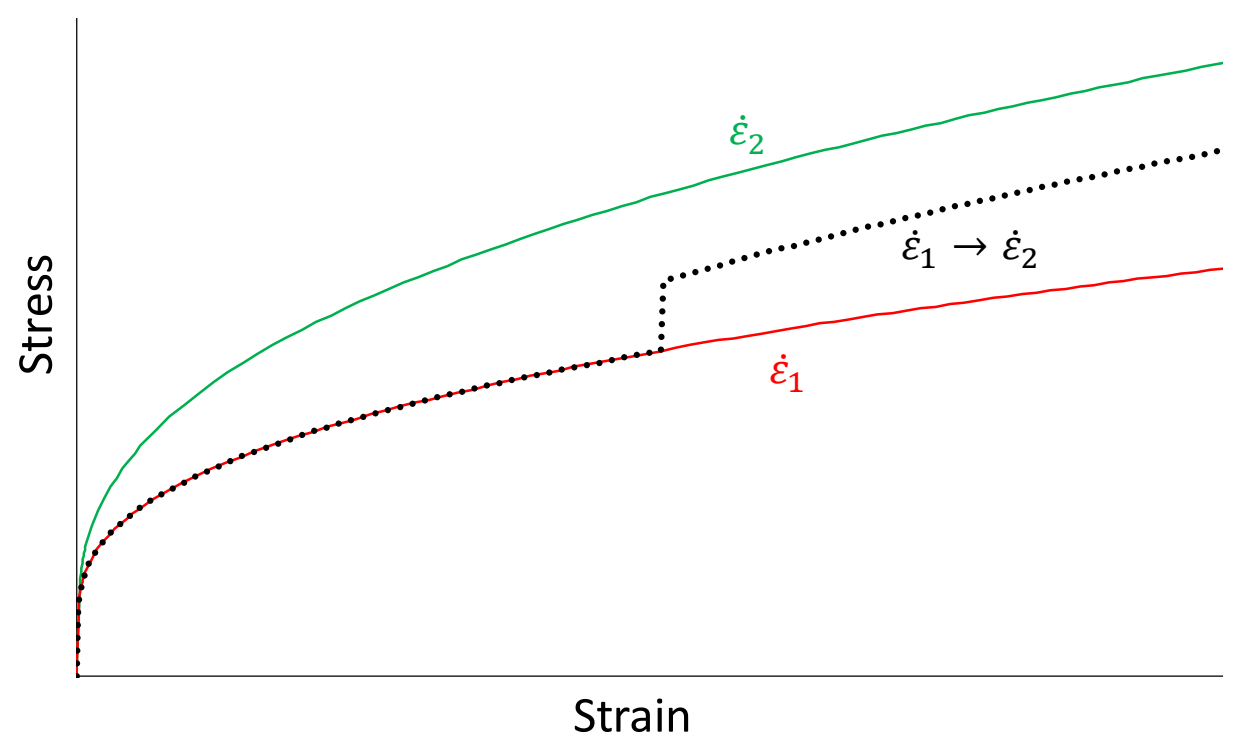

Fig. 1. Schematic stress-strain curves illustrating strain rate and strain-rate history effects for FCC and HCP materials (adapted from the experimental results presented in (Klepaczko, 1975) and (Senseny et al., 1978)). 
Strain-rate history effects are related to the fact that the microstructural evolution of a material with the deformation may be dependent on the applied strain rate $^{1}$. For instance, Chiem and Duffy (1983) carried out observations of the dislocation substructure in single-crystal aluminium specimens deformed at quasi-static and high strain rates and detected that for a given strain level the dislocation density is larger when the material is deformed at high strain rate. Therefore, for materials with strain-rate history effects, it is necessary to distinguish the instantaneous strain-rate sensitivity (related to the dependence of flow stress on strain rate at given microstructure) and the strain-rate sensitivity of work hardening (related to the influence of strain rate on the microstructure evolution). The strain-rate sensitivity of work hardening is particularly marked for FCC metals (Klepaczko and Chiem, 1986; Lea, 2018).

A variety of constitutive models have been proposed to describe strain-rate history effects (Estrin and Mecking, 1984; Klepaczko and Chiem, 1986; Follansbee and Kocks, 1988; Tong et al., 1992; Bodner and Rubin, 1994; Molinari and Ravichandran, 2005; dos Santos et al., 2016; Lea, 2018). These models can be either based on physical considerations (e.g. dislocation density evolution) or phenomenological. However, a common feature of these models is that hardening is described by an internal mnesic variable whose evolution is strain-rate dependent. Walley et al. (2000) presented a comparison between experimental results of Taylor-impact tests on XM copper and numerical simulations based on two different constitutive models: the standard Zerilli-Armstrong (1987) viscoplastic relation and an extended version of this model which takes strain-rate history effects into account (Gould and Goldthorpe, 2000). It was found that using the history-dependent model significantly improves the accuracy of the numerical results. This shows that accounting for strain-rate history effects may be important to describe the response of structures under dynamic loading. As mentioned at the beginning of this introduction, the predictions of plastic flow instabilities are generally highly dependent on the constitutive model assumed, see for e.g. (Xue et al., 2008; Vadillo et al., 2012; Jacques, 2020). Therefore, one may wonder what is the role played by strain-rate history effects in dynamic necking problems and what are the relative contributions of the instantaneous and workhardening strain-rate sensitivities. These questions, that to the best of the authors' knowledge have not been addressed in the literature, are the subject of the present study. Two different problems are considered: the impact tension test and the ring expansion test. As mentioned

\footnotetext{
${ }^{1}$ Note that the microstructure evolution may be also temperature dependent (Klepaczko and Chiem, 1986).
} 
previously, there is an important difference between these two problems: owing to the radial symmetry of the ring expansion test, the deformation state in the specimen is nearly homogeneous until multiple necks are nucleated in the circumference of the specimen, whereas the tension impact test is generally affected by wave propagation effects, leading to nonhomogeneous strain and stress fields (Hu and Daehn, 1996). The two problems are investigated using finite element simulations in which the material is modelled with a constitutive relation that accounts for strain-rate history effects. Moreover, an analytical model (linear stability analysis) is developed for the ring expansion problem.

\section{A constitutive model with strain-rate history effects}

In this section, an elastic-viscoplastic model with strain-rate history effects is presented. As mentioned in the introduction, several models taking strain-rate history effects into account have been proposed in the literature, see for e.g. (Estrin and Mecking, 1984; Klepaczko and Chiem, 1986; Follansbee and Kocks, 1988; Tong et al., 1992; Bodner and Rubin, 1994; Molinari and Ravichandran, 2005; dos Santos et al., 2016, Lea, 2018). However, we have developed a specific constitutive relation for the present study. This model is well-suited for parametric studies as the relative contribution of instantaneous strain-rate sensitivity and workhardening strain-rate sensitivity to material response is controlled by a single parameter. Moreover, the model has been specifically designed to facilitate the comparison of the necking behaviour of different materials having the same stress-strain response at constant strain rate, but different strain-rate history effects.

The constitutive framework relies on the classical assumption of the additive decomposition of the rate of deformation tensor and the $J_{2}$ flow theory. The total rate of deformation tensor $\boldsymbol{d}$ is the sum of an elastic and a plastic part:

$$
\boldsymbol{d}=\boldsymbol{d}^{e}+\boldsymbol{d}^{p}
$$

The elastic rate of deformation $\boldsymbol{d}^{e}$ is related to the Cauchy stress tensor $\boldsymbol{\sigma}$ by a hypoelastic relation of the form

$$
\widetilde{\boldsymbol{\sigma}}=\frac{E}{1+v}\left[\boldsymbol{d}^{e}+\frac{v}{1-2 v} \operatorname{tr}\left(\boldsymbol{d}^{e}\right) \mathbf{1}\right]
$$


where $\widetilde{\boldsymbol{\sigma}}$ is an objective time derivative of the Cauchy stress tensor, $E$ and $v$ are the Young modulus and the Poisson ratio, respectively, $\mathbf{1}$ is the second order unit tensor and tr denotes the trace operator. The plastic rate of deformation is given by the $J_{2}$ flow law:

$$
\boldsymbol{d}^{p}=\frac{3}{2} \dot{\bar{\varepsilon}} \frac{\boldsymbol{s}}{\sigma_{e}}
$$

where $\boldsymbol{s}$ is the deviatoric stress tensor, $\boldsymbol{s}=\boldsymbol{\sigma}-\frac{1}{3} \operatorname{tr}(\boldsymbol{\sigma}) \mathbf{1}, \sigma_{e}$ is the von Mises effective stress, $\sigma_{e}=\sqrt{\frac{3}{2} \boldsymbol{s}: \boldsymbol{s}}$, and $\dot{\bar{\varepsilon}}$ is the effective plastic strain rate, $\dot{\bar{\varepsilon}}=\sqrt{\frac{2}{3} \boldsymbol{d}^{p}: \boldsymbol{d}^{p}}$.

The yield function $f$ associated to the model is

$$
f=\sigma_{e}-\bar{\sigma}(H, \dot{\bar{\varepsilon}})
$$

where $\bar{\sigma}$ is the flow stress of the material

$$
\bar{\sigma}(H, \dot{\bar{\varepsilon}})=\sigma_{0}+H \cdot(1+(1-\alpha) \cdot z(\dot{\bar{\varepsilon}})) \cdot \omega(\dot{\bar{\varepsilon}})
$$

The functions $z(\dot{\bar{\varepsilon}})$ and $\omega(\dot{\bar{\varepsilon}})$ are defined as

$$
\begin{aligned}
& z(\dot{\bar{\varepsilon}})=K \cdot\left(\frac{\dot{\bar{\varepsilon}}}{\dot{\varepsilon}_{R}}\right)^{m} \\
& \omega(\dot{\bar{\varepsilon}})=\sqrt{\frac{1+z(\dot{\bar{\varepsilon}})}{1+z(\dot{\bar{\varepsilon}})+\alpha(1-\alpha) z(\dot{\bar{\varepsilon}})^{2}}}
\end{aligned}
$$

In Eqs. (5)-(7), $\sigma_{0}, K, m, \dot{\varepsilon}_{R}$ and $\alpha$ are constants, while $H$ is the hardening variable. The evolution of $H$ is strain-rate dependent:

$$
\frac{\partial H}{\partial \bar{\varepsilon}}=\sigma_{R} \cdot n \cdot(\bar{\varepsilon})^{n-1} \cdot(1+\alpha \cdot z(\dot{\bar{\varepsilon}})) \cdot \omega(\dot{\bar{\varepsilon}})
$$

with $\bar{\varepsilon}=\int_{0}^{t} \dot{\bar{\varepsilon}}$. $\mathrm{d} \tau$ being the effective plastic strain. Moreover, $\sigma_{R}$ is a reference stress and $n$ is the hardening exponent. In the following, we will assume the initial value of the hardening variable is equal to zero $(H=0$ for $\bar{\varepsilon}=0)$. Eq. (8) describes the strain-rate sensitivity of work hardening. Indeed, it is apparent from Eq. (8) that the value of $H$ at a given time (or strain level) depends on the preceding strain-rate evolution.

Next, let us discuss the role played by two key features of the constitutive model: the function $z(\dot{\bar{\varepsilon}})$ and the parameter $\alpha$ (see Eqs. (5)-(8)). The function $z(\dot{\bar{\varepsilon}})$ characterises the global (or overall) strain-rate sensitivity of the material. By global, we mean the rate-sensitivity observed 
from several tests performed at constant strain rate. Indeed, when $\dot{\bar{\varepsilon}}$ is constant with $H=0$ for $\bar{\varepsilon}=0$, the flow stress can be expressed in the following form:

$$
\bar{\sigma}=\sigma_{0}+\sigma_{R} \cdot \bar{\varepsilon}^{n} \cdot(1+z(\dot{\bar{\varepsilon}}))
$$

The parameter $\alpha$ characterises strain-rate history effets. Its influence is illustrated in Fig. 2, which displays the response to a plastic strain-rate jump (from $\dot{\bar{\varepsilon}}_{1}$ to $\dot{\bar{\varepsilon}}_{2}$ ) for several values of $\alpha$. For $\alpha=0$, the hardening variable $H$ can be expressed as a function of the effective plastic strain, $H=\sigma_{R} \cdot \bar{\varepsilon}^{n}$. Therefore, the flow stress depends on the current values of the plastic strain and strain-rate (but not on the previous strain rate history). The material does not have rate history effects or, in other words, only shows instantaneous strain-rate sensitivity. Consequently, the stress after the strain-rate jump is the same as if the material had always been deformed at $\dot{\bar{\varepsilon}}_{2}$ (Fig. 2). On the other hand, for $\alpha=1$, the flow stress is no longer dependent on the current value of the plastic strain rate (the expression of the flow stress reduces to $\bar{\sigma}=\sigma_{0}+$ $H$ ). The rate sensitivity of the material response is only due to the influence of strain rate on the evolution of its microstructure. The material has pure work-hardening strain-rate sensitivity (or equivalently no instantaneous strain-rate sensitivity). In this case, a plastic strain-rate jump does not lead to a jump of the flow stress (Fig. 2). Intermediate values of $\alpha$ correspond to materials with both instantaneous strain-rate sensitivity and work-hardening strain-rate sensitivity. A sudden increase in plastic strain rate causes a flow stress jump, whose magnitude is proportional to $1-\alpha$.

The present elastic-viscoplastic model has been implemented in the finite element software ABAQUS/Explicit. For this purpose, a user material subroutine (VUMAT) has been developed. The numerical integration of the constitutive equations is based on the radial return algorithm, see for e.g. (Simo and Hughes, 1998). 


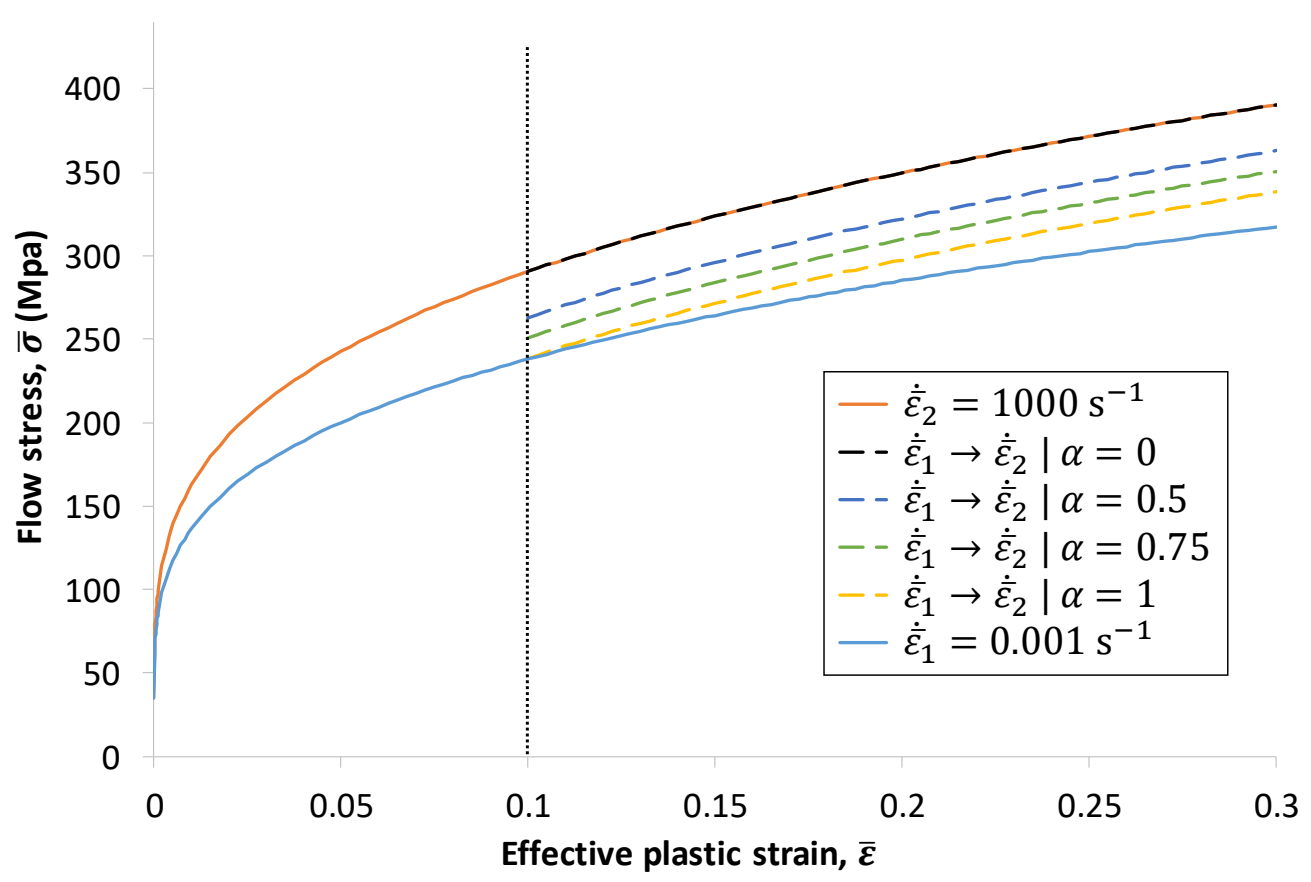

Fig. 2. Illustration of the role of the parameter $\alpha$ of the constitutive model presented in section 2. The solid lines show the stress-strain curves for constant values of plastic strain-rate, $\dot{\bar{\varepsilon}}_{1}=0.001 \mathrm{~s}^{-1}$ and $\dot{\bar{\varepsilon}}_{2}=1000 \mathrm{~s}^{-1}$. The dashed lines show the stress evolution caused by a plastic strain-rate jump from $\dot{\bar{\varepsilon}}_{1}$ to $\dot{\bar{\varepsilon}}_{2}$ at $\bar{\varepsilon}=0.1$ for several values of parameter $\alpha$, see Eqs. (5), (8). Other parameters are given in table 1.

\begin{tabular}{|c|c|c|}
\hline Young modulus & $E$ & $124000 \mathrm{MPa}$ \\
\hline Poisson ratio & $v$ & 0.34 \\
\hline Initial mass density & $\rho_{0}$ & $8960 \mathrm{~kg} / \mathrm{m}^{3}$ \\
\hline Initial yield stress & $\sigma_{0}$ & $35 \mathrm{MPa}$ \\
\hline Reference stress & $\sigma_{R}$ & $300 \mathrm{MPa}$ \\
\hline Hardening exponent & $n$ & 0.3 \\
\hline Reference strain rate & $\dot{\varepsilon}_{R}$ & $1000 \mathrm{~s}^{-1}$ \\
\hline Strain-rate sensitivity exponent & $m$ & 0.05 \\
\hline Strain-rate sensitivity coefficient & $K$ & 0.7 \\
\hline Strain-rate history effect parameter & $\alpha$ & Ranging between 0 and 1 \\
\hline
\end{tabular}

Table 2. Material parameters representative of copper used in the numerical simulations and the linear stability analysis. 


\section{Round bar under impact tension}

\subsection{Finite element model}

Lagrangian finite element computations have been performed using ABAQUS/Explicit (version 2017) to analyse the influence of strain-rate history effects on neck formation in a linear specimen with circular cross-section subjected to dynamic uniaxial tensile loading. The geometry and the dimensions of the specimen are shown in Fig. 3. The finite element model developed in ABAQUS/Explicit is axisymmetric, and the mesh consists of 14250 quadrilateral elements with bilinear interpolation functions, reduced integration and hourglass control (CAX4R in ABAQUS notation). The element size in the gauge of the specimen is equal to $40 \times 30 \mu \mathrm{m}^{2}$ (the largest value being the element dimension in the radial direction).

The specimen is initially at rest:

$$
V_{R}(R, Z, 0)=V_{Z}(R, Z, 0)=0, \quad \boldsymbol{\sigma}(R, Z, 0)=\mathbf{0}, \quad H(R, Z, 0)=0
$$

where $V_{R}$ and $V_{Z}$ are the radial and axial components of the velocity vector, respectively, $\boldsymbol{\sigma}$ is the Cauchy stress tensor and $H$ is the hardening variable used in the constitutive model (see section 2).

The bottom surface of the specimen is clamped:

$$
V_{R}(R, 0, t)=V_{Z}(R, 0, t)=0
$$

On the top surface, a constant velocity is applied in the axial direction:

$$
V_{Z}(R, 0, t)=V_{i m p}, \quad V_{R}(R, 0, t)=0
$$

where $V_{i m p}$ is the magnitude of the applied velocity, hereinafter also referred to as the impact velocity. 

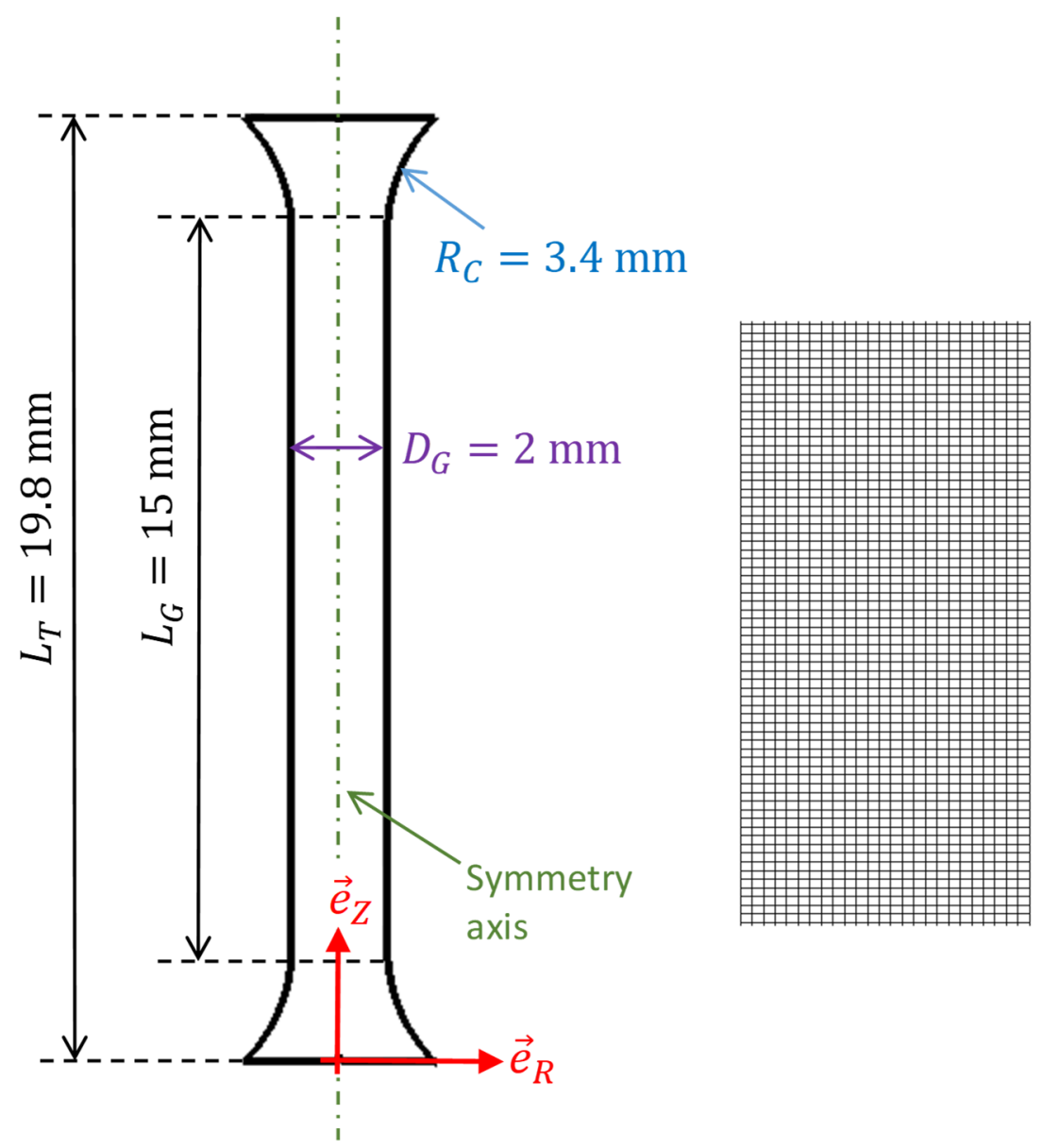

Fig. 3. Geometry of the specimen considered in the simulations of impact tests presented in section 3. The figure also shows a close view of the mesh in the gauge region (note that only the part on the right side of the symmetry axis is modelled in ABAQUS).

\subsection{Results}

Fig. 4 presents the deformed geometry of the specimen and the distribution of effective plastic strain obtained for an impact velocity of $20 \mathrm{~m} / \mathrm{s}$ and three values of the parameter $\alpha$ that characterises strain-rate history effects: $\alpha=0$ (pure instantaneous strain-rate sensitivity), $\alpha=$ 0.5 and $\alpha=1$ (pure work-hardening strain-rate sensitivity), see also Fig. 2. For each value of $\alpha$, the plastic strain distribution presented in Fig. 4 corresponds to a time such that the plastic strain at the centre of the neck is close to 1.5. This value was chosen because the neck is fully formed (plastic deformation concentrates in the necked region) for the three values of $\alpha$. Moreover, this value is close to the fracture strain of annealed copper under uniaxial tension (French and Weinrich, 1975). Hereinafter, the time for which the plastic strain at the neck centre reaches 1.5 will be referred to as the localisation time. It is apparent from Fig. 4 that the elongation of the specimen at the localisation time depends on the value of $\alpha$ used in the 
simulations. The largest elongation is obtained for the material having a pure instantaneous strain-rate sensitivity $(\alpha=0)$. When strain-rate history effects are taken into account $(\alpha=0.5$ and $\alpha=1$ ), the localisation time and the corresponding elongation of the specimen are significantly reduced. These results suggest that the formation of the neck is affected by strainrate history effects. To further illustrate this point, Fig. 5 displays the time history of the plastic strain (a) and the plastic strain-rate (b) at the neck centre for several values of $\alpha$ and for a rateindependent material (i.e. when the strain-rate sensitivity coefficient is $K=0$, see Eq. (6)). In Fig. 5-a, we have also plotted the strain evolution calculated assuming that the strain in the gauge of the specimen is homogeneous (generally referred to as homogeneous deformation solution). The neck formation leads to a deviation of the strain from the homogenous deformation solution and a rapid increase in strain rate in the necked region. When the value $\alpha$ is decreased, the deviation of the plastic strain evolution from the homogenous solution is delayed. Moreover, notice that the results obtained with $\alpha=1$ are rather close to those obtained with the rate-independent material. Fig. 5-a also shows the instability strain associated to the maximum force Considère criterion of the rate-dependent materials ${ }^{2}$. Recall that the Considère criterion characterises the onset of necking in rate-independent materials under uniaxial tension. We observe that for the material with a pure work-hardening strain-rate sensitivity $(\alpha=1)$ the necking instability develops rapidly when the Considère strain is met. This result is consistent with the force evolutions presented in Fig. 6. With $\alpha=1$, the force drops rapidly after it reaches the peak value. When the value of $\alpha$ is decreased (corresponding to greater contribution of the instantaneous strain-rate sensitivity), the formation of the neck and the resulting force drop are significantly delayed.

As discussed in the introduction, for materials that exhibit strain-rate history effects, it is important to distinguish between the instantaneous strain-rate sensitivity and the workhardening strain-rate sensitivity. From the results presented in Figs. 5 and 6, it appears that only the instantaneous strain-rate sensitivity leads to neck retardation effect. When the relative contribution of the work-hardening strain-rate sensitivity is increased, the neck retardation effect is significantly decreased (for the same global strain-rate sensitivity). For the material with a pure work-hardening strain-rate sensitivity $(\alpha=1)$, the development of the necking instability is very close to that of the rate-independent material.

\footnotetext{
${ }^{2}$ The Considère strain was computed using the condition $\frac{\partial \bar{\sigma}}{\partial \bar{\varepsilon}}=\bar{\sigma}$ and considering the initial strain-rate. It is equal to 0.273 , irrespectively of the value of $\alpha$.
} 


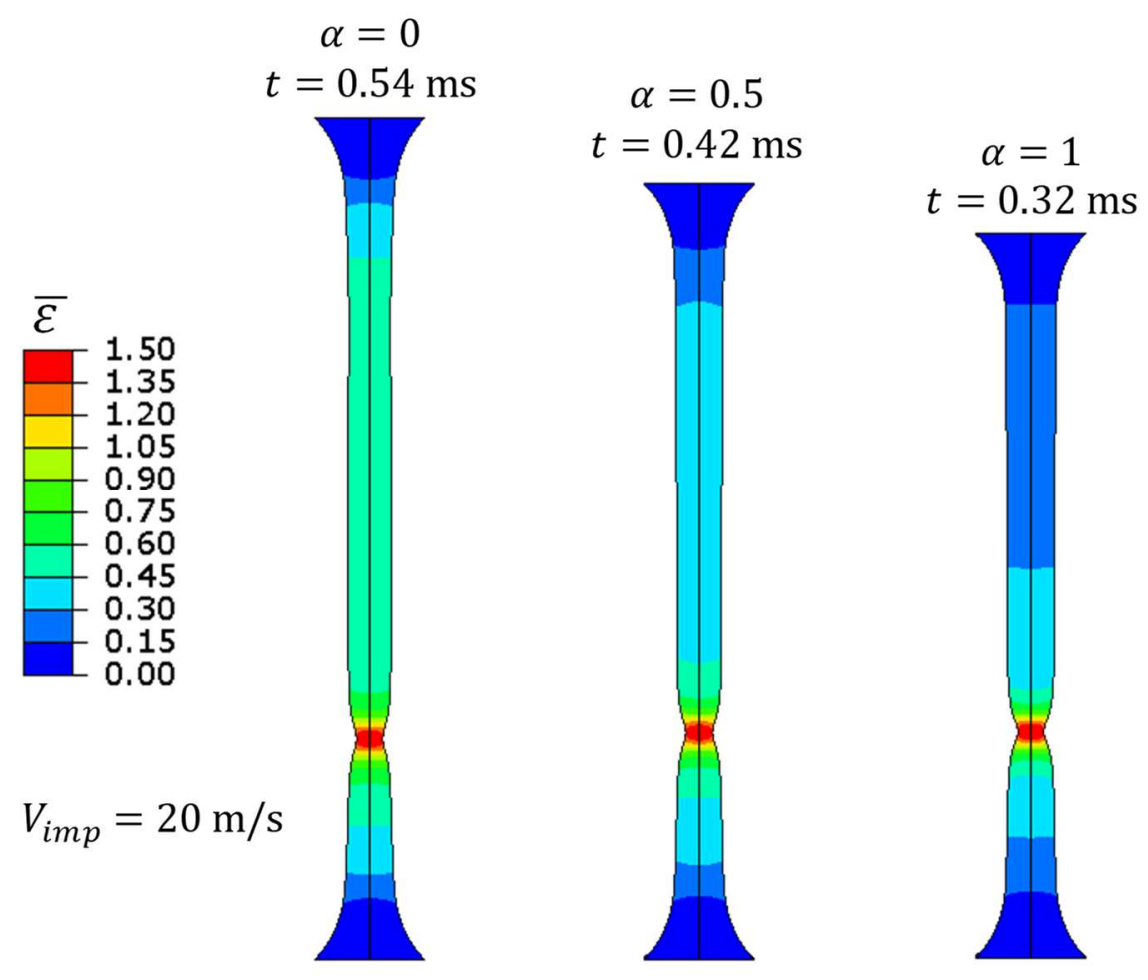

Fig. 4. Deformed geometry of the bar specimen and distribution of plastic strain for several values of the strain-rate history effect parameter $\alpha$ and an impact velocity $V_{i m p}=20 \mathrm{~m} / \mathrm{s}$. For each value of $\alpha$, the time is chosen so that the maximum effective plastic strain is $\bar{\varepsilon} \sim 1.5$. Other material parameters are given in table 1.

(a) 


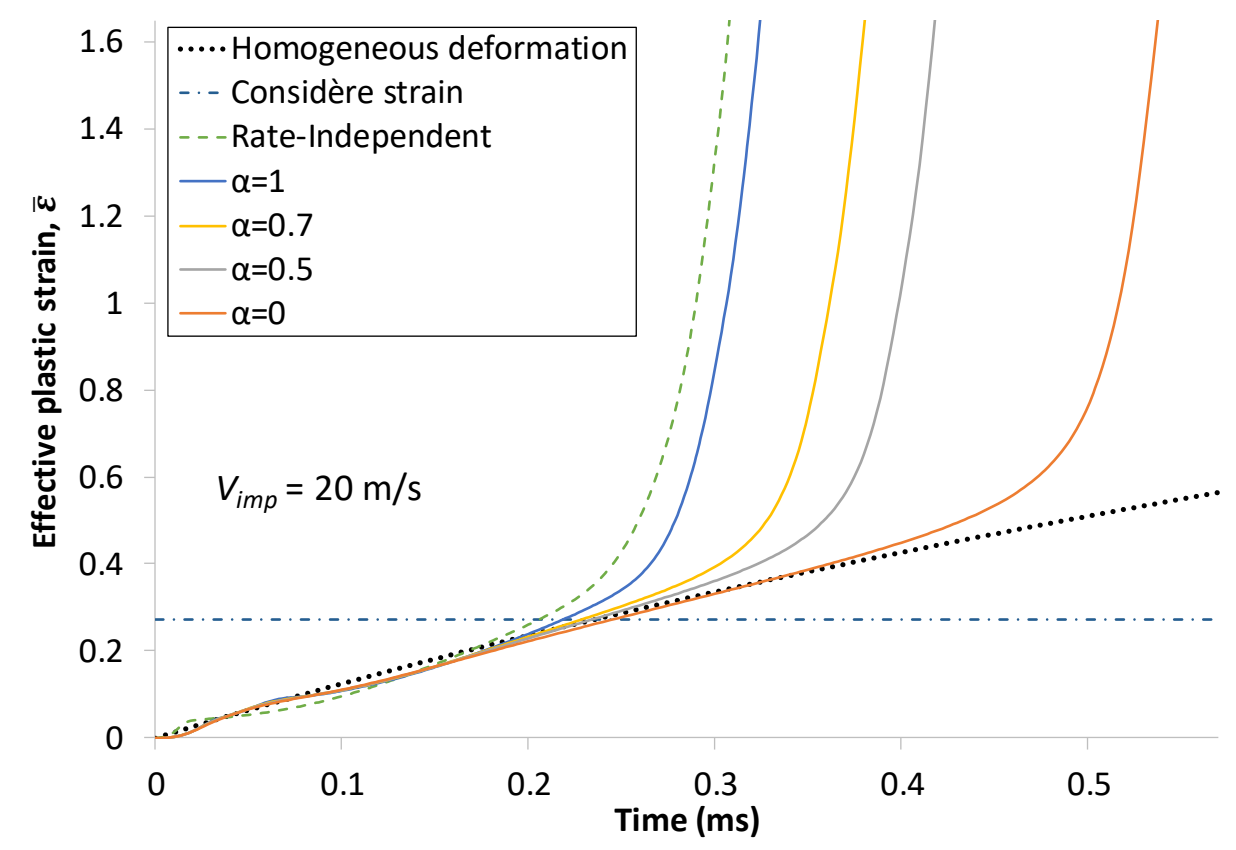

(b)

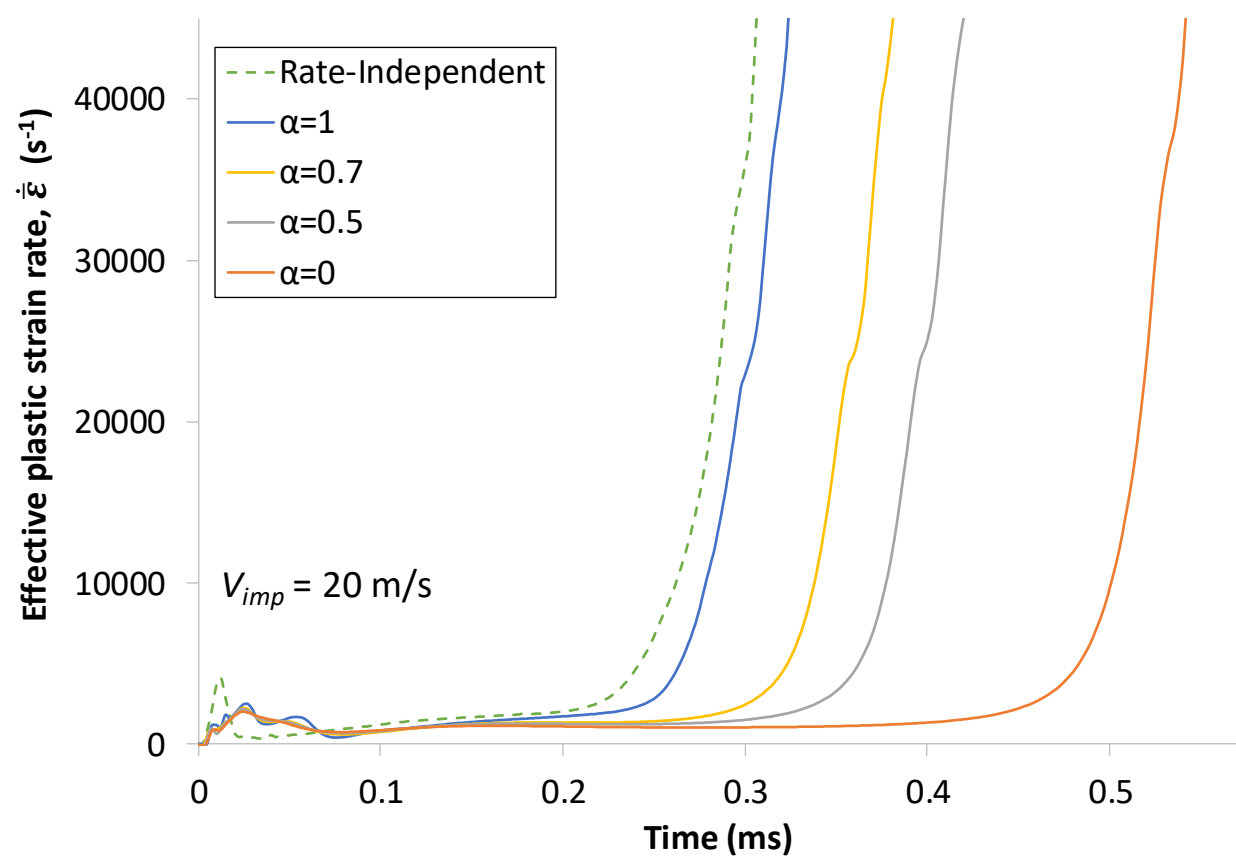

Fig. 5. Evolution of the (a) effective plastic strain and (b) effective plastic strain rate at the centre of the neck for several values of the strain-rate history effect parameter $\alpha$ and an impact velocity $V_{i m p}=$ $20 \mathrm{~m} / \mathrm{s}$. The results obtained for a rate-independent material ( $K=0$ in Eqs. (6)) are also presented. In Fig. 5-a, both the strain evolution obtained assuming a homogeneous deformation in the gauge region of the specimen and the Considère strain (for the rate-dependent materials) are shown. Other material parameters are given in table 1. 


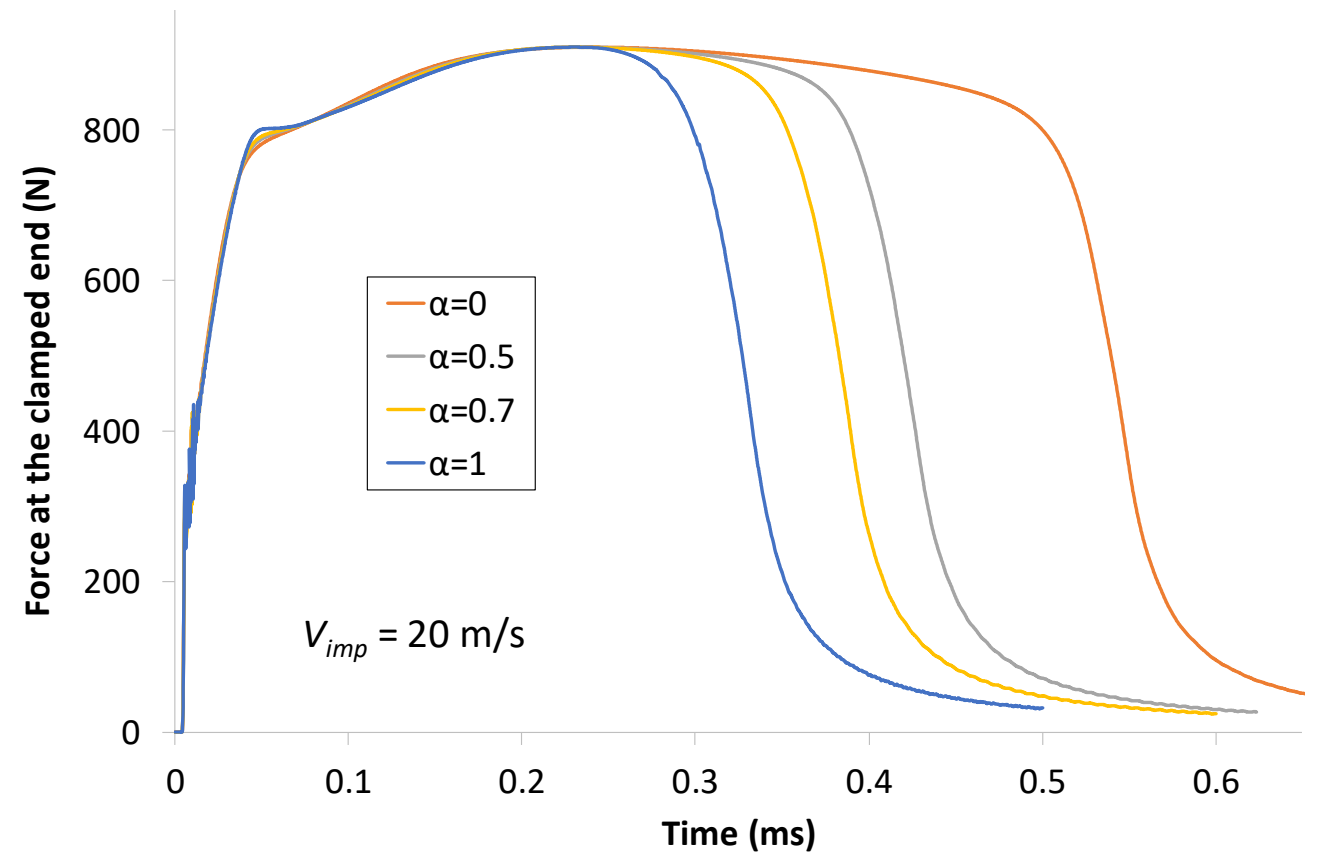

Fig. 6. Evolution of the axial force acting on the clamped surface of the specimen for several values of the strain-rate history effect parameter $\alpha$ and an impact velocity $V_{\text {imp }}=20 \mathrm{~m} / \mathrm{s}$. Other material parameters are given in table 1 .

Fig. 7-a presents the evolution of the effective stress with the plastic strain at the centre of the neck obtained for several values of the strain-rate history effect parameter $\alpha$ and an impact velocity of $20 \mathrm{~m} / \mathrm{s}$. For comparison purpose, a reference stress-strain curve corresponding to a constant plastic strain rate of $1666 \mathrm{~s}^{-1}$ (the nominal strain rate $\left(V_{\text {imp }} / L_{G}\right)$ for the considered applied velocity) is also plotted. The Considère strain of the materials is also displayed. Some differences between the curves obtained with the different values of $\alpha$ are observed for large strains, higher than the Considère strain. At these strain levels, the strain is no longer homogeneous along the gauge length of the specimen. The necking development leads to a concentration of the strain and an increase in strain rate inside the necked region. In fact, the differences between the stress-strain responses measured at the centre of the neck and this reference curve are related to the combined effect of this increase in strain rate caused by the formation of the neck and the strain-rate sensitivity of the different materials. The evolution of the effective plastic strain rate at the neck centre as a function of the plastic strain is shown in Fig. 7-b. It is interesting to observe that for a given value of plastic strain, the strain rate is larger when the parameter $\alpha$ is increased (corresponding to more pronounced strain-rate history effects) as the necking instability develops faster. However, the resulting stress increase is lower. When $\alpha$ is increased, the role of the work-hardening strain-rate sensitivity becomes 
predominant. Contrary to the instantaneous strain-rate sensitivity, the work-hardening strainrate sensitivity is a cumulative mechanism. Its influence on the mechanical response of the material does not only depends on the current value of the strain rate, but on the previous strain rate history. For this reason, a rapid increase in strain rate does not immediately leads to an increase in the stress level. This is probably the reason why the work-hardening strain-rate sensitivity does not significantly slow down the formation of the neck (contrary to the instantaneous strain-rate sensitivity).

It is interesting to draw a parallel between the results presented in Fig. 7 and the locking phenomenon reported in recent experimental studies (Mirone et al., 2019, 2020). These authors observed from split Hopkinson bar tensile tests that even if the strain-rate strongly increases after the onset of necking, this steep increase does not cause a significant amplification of the flow stress. This locking phenomenon could be related to strain-rate history effects.

(a)

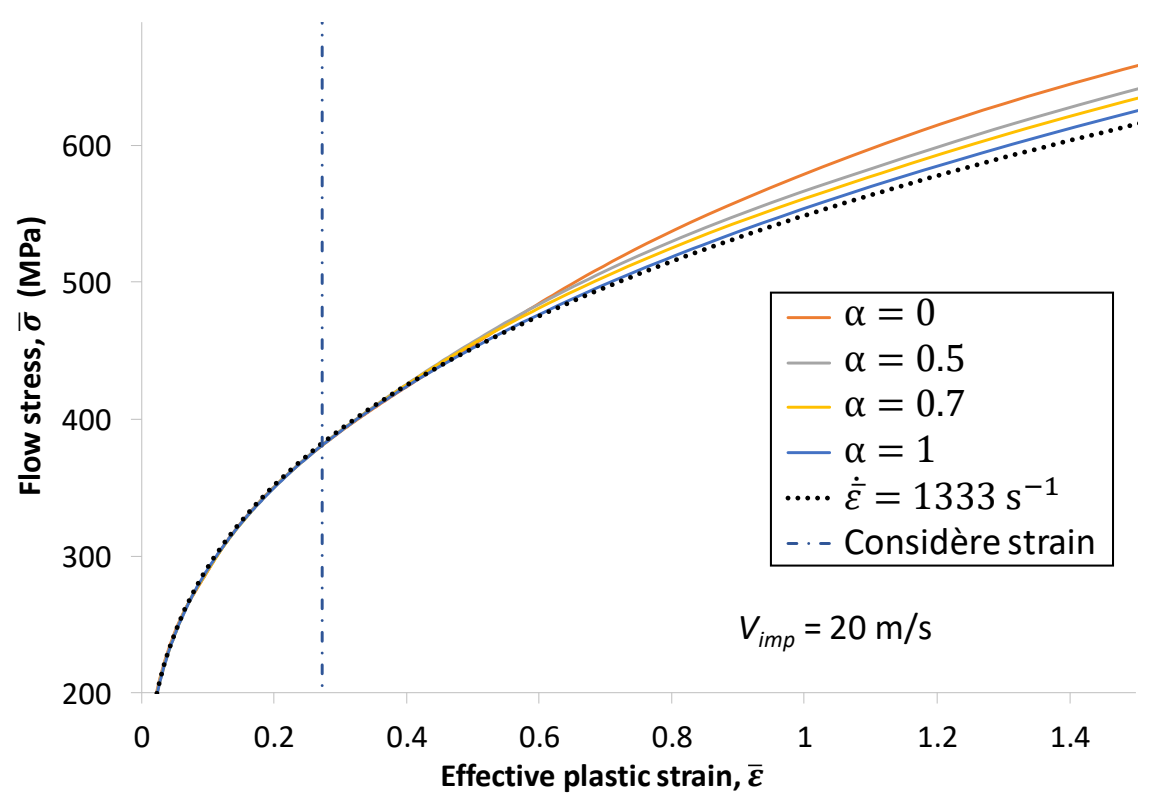

(b) 


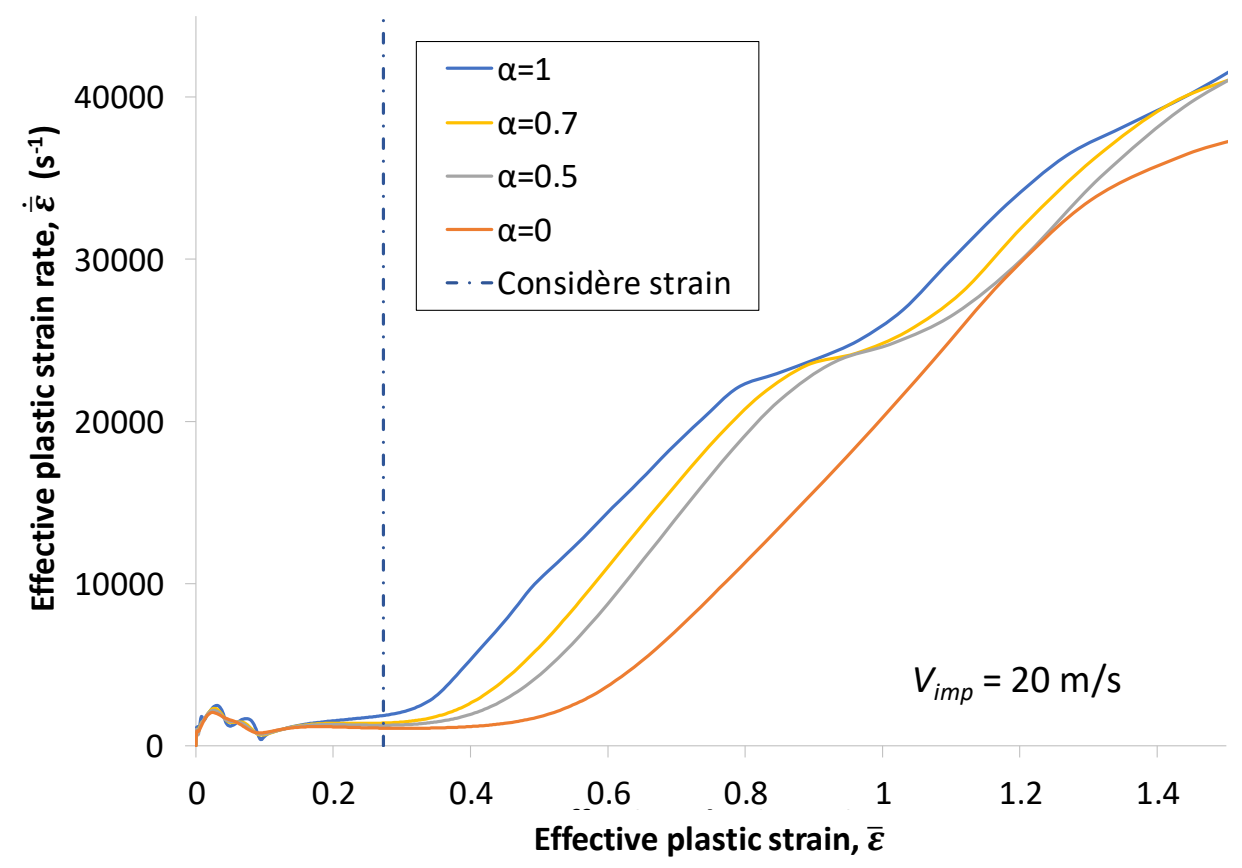

Fig. 7. (a) Flow stress versus effective plastic at the centre of the neck for several values of the strainrate history effect parameter $\alpha$. For comparison purpose, the stress-strain curve for a constant strain rate of $1666 \mathrm{~s}^{-1}$ (corresponding to the nominal strain rate for the considered impact velocity) and the Considère strain are also displayed. The beginning of the stress-strain curves (for stresses lower than $200 \mathrm{MPa}$ ) is not represented to make the differences between the curves more visible. (b) Effective plastic strain rate versus effective plastic strain relation observed at the centre of the neck for several values of strain-rate history effect parameter $\alpha$. The applied velocity is $V_{i m p}=20 \mathrm{~m} / \mathrm{s}$. Other material parameters are given in table 1.

We now investigate the influence of the applied velocity. Figs. 8-a and 8-b present respectively the evolution of the normalised localisation time (defined as $\hat{t}_{l o c}=V_{i m p} t_{l o c} / L_{G}$ with $t_{l o c}$ being the time for which the peak plastic strain reaches 1.5) and the energy dissipated at localisation as a function of the impact velocity for several values of strain-rate history effect parameter $\alpha$. One notices that both quantities follow the same evolution and two different regimes are apparent. When the applied velocity is lower than $\sim 70 \mathrm{~m} / \mathrm{s}$, the normalised localisation time and the dissipated energy show little dependence on the applied velocity. Moreover, in this range of velocities, the localisation time and the energy dissipated at localisation are both significantly affected by the nature of strain-rate sensitivity effects. They are maximal for $\alpha=0$ (pure instantaneous rate sensivity) and decrease significantly for larger values of $\alpha$. This finding is consistent with the observations made previously that showed that only the instantaneous strainrate sensitivity delays necking formation. However, we observe that the differences between 
the localisation time and energy obtained for the three values of $\alpha$ tend to decrease gradually with increasing impact velocity. When the velocity exceeds $\sim 70 \mathrm{~m} / \mathrm{s}$, both the localisation time and the energy drop rapidly and become independent of the value of $\alpha$, so that they are no longer influenced by the strain-rate history effects. This velocity of $\sim 70 \mathrm{~m} / \mathrm{s}$ is the critical impact velocity (CIV) that according to Mann (1936), Clark and Wood (1950) and Klepaczko (2005), among others, determines the maximum energy absorbed by the sample and the maximum sample ductility (which corresponds to the maximum normalised localisation time) in the dynamic tension test. The CIV also leads to a change of the mode of localisation, see Rusinek et al. (2005), so that for $V_{i m p} \leq$ CIV localisation is induced by a plastic flow instability (leading to a gradual destabilisation of a nearly homogeneous deformation state), while for $V_{\text {imp }}>\mathrm{CIV}$, localisation is due to plastic wave trapping (von Karman and Duwez, 1950; Klepaczko, 2005). In this case, localisation takes place near the impacted end of the bar right after the application of the loading, the rest of the bar remaining virtually free of plastic deformation, see Fig. 9. A very interesting observation from Fig. 8 is that the critical impact velocity is roughly the same for the three materials ( $\alpha=0, \alpha=0.5$ and $\alpha=1$ ), meaning that it is largely insensitive to strain-rate history effects. This phenomenon is related to the fact the propagation of plastic waves through the specimen is almost unaffected by strain-rate history effects (see infra).

Fig. 8-c shows that the value of the rate history effect parameter $\alpha$ generally does not have a significant influence on the location of the main neck. It has been shown in the literature that the location of necking in linear specimens under impact loading is controlled by the mechanisms of wave propagation, reflection and interaction in the sample (Clark and Duwez, 1948; Nemes and Eftis, 1993; Osovki et al., 2013; Vaz-Romero et al., 2015). From the results of the finite element computations, we have observed that strain-rate history effects have little influence on the propagation of plastic waves in the specimen (results not presented). This explains why neck location is almost unaffected by strain-rate history effects (or in other words by the relative contributions of the instantaneous and work-hardening strain-rate sensitivities). 
(a)

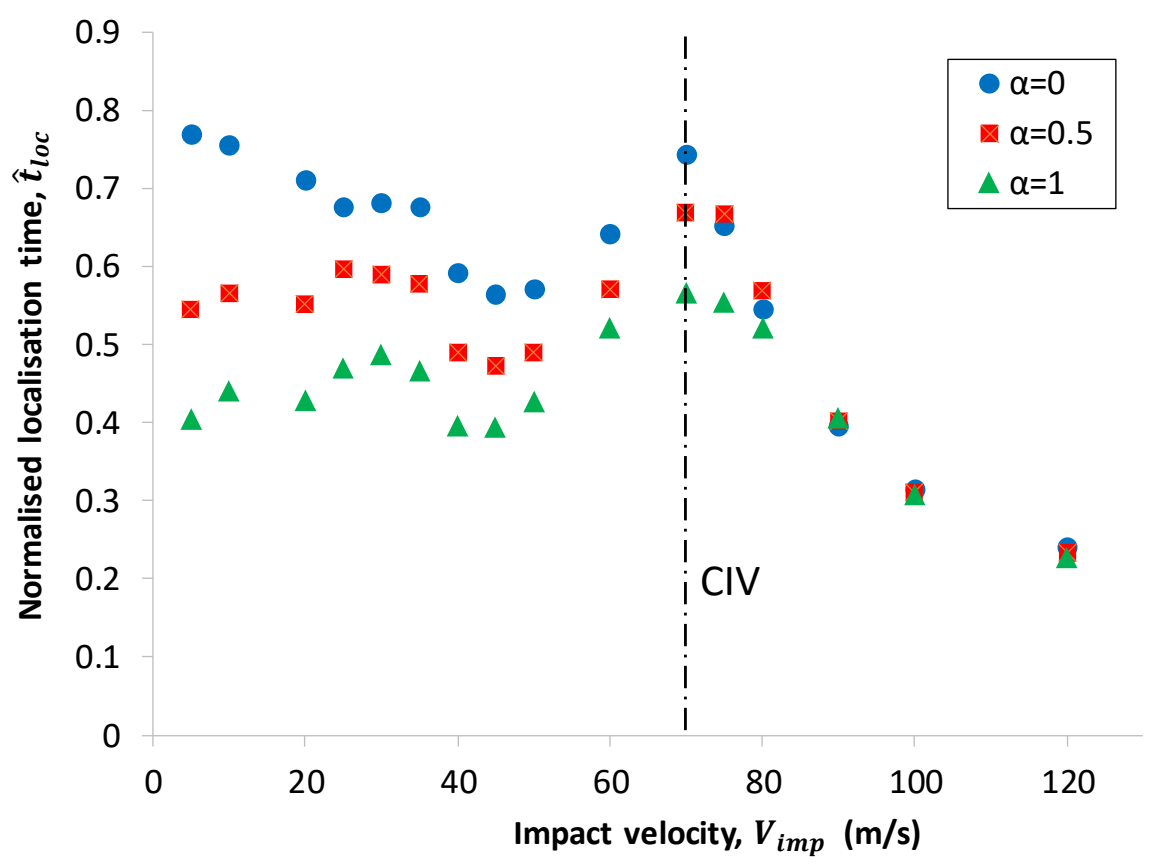

(b)

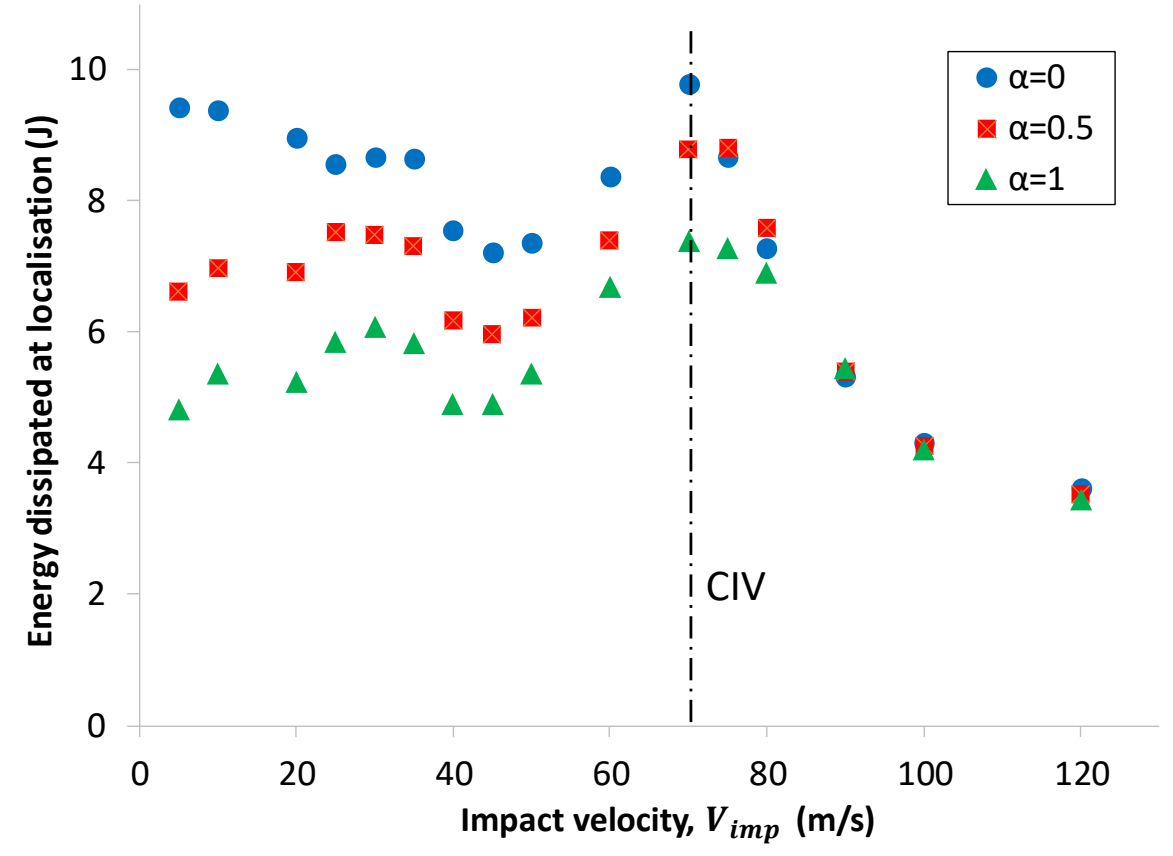

(c) 


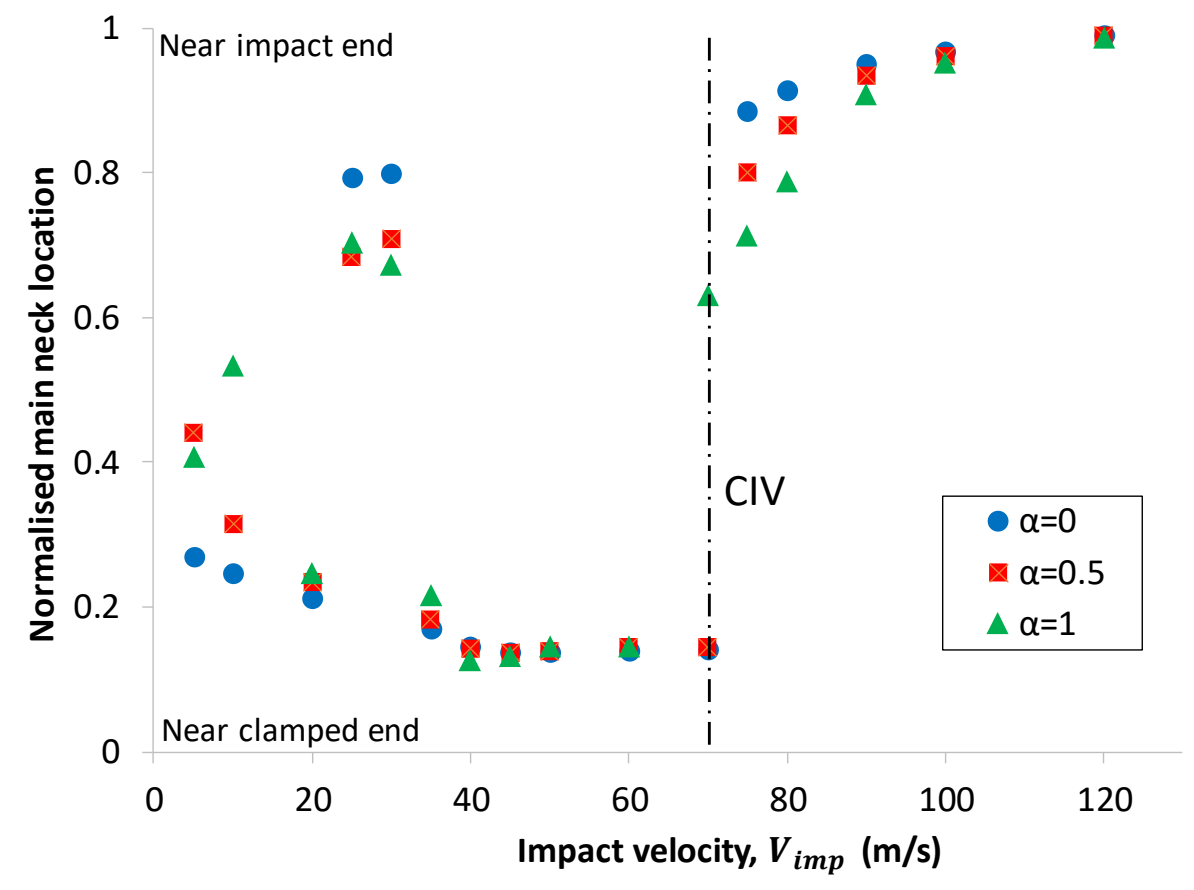

Fig. 8. Evolution of (a) the normalised localisation time, (b) the energy dissipated at localisation and (c) the normalised Lagrangian neck location as a function of the impact velocity for several values of the strain-rate history effect parameter $\alpha$. Other material parameters are given in table 1.

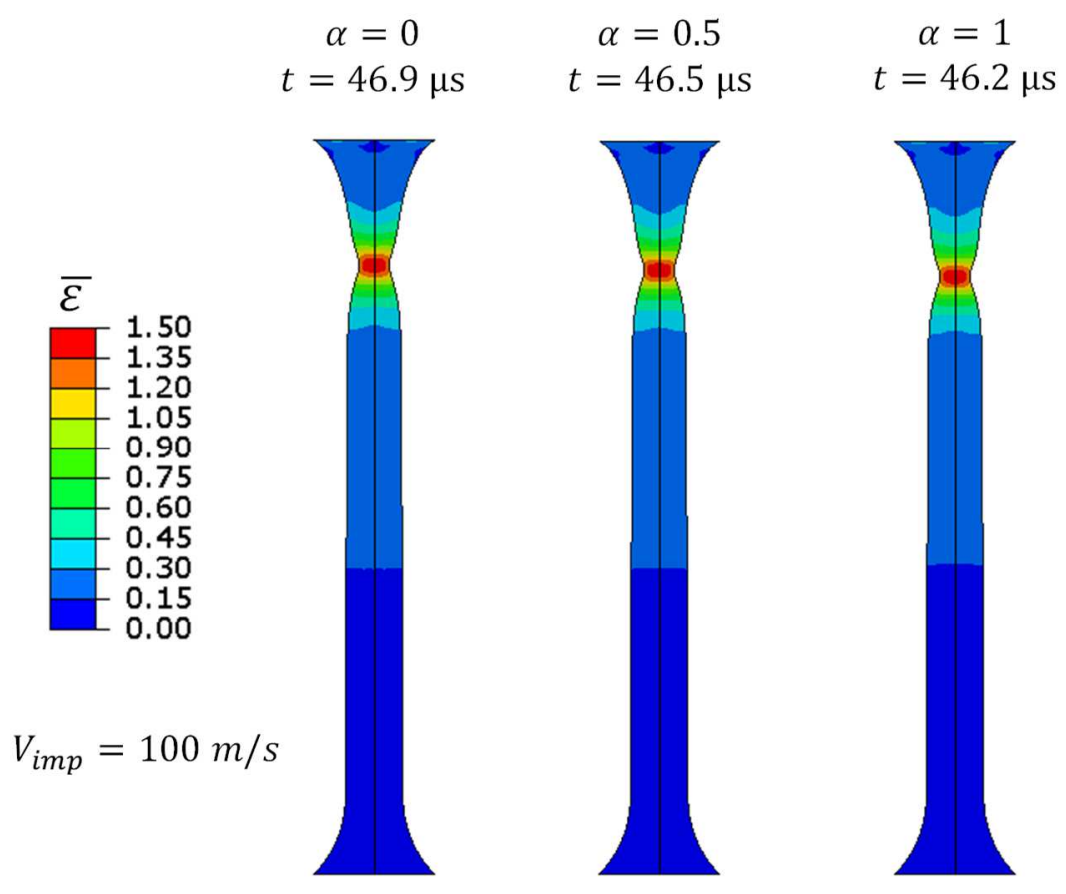

Fig. 9. Deformed geometry of the bar specimen and distribution of plastic strain for several values of the strain-rate history effect parameter $\alpha$ and an impact velocity $V_{\text {imp }}=100 \mathrm{~m} / \mathrm{s}$. For each value of 
$\alpha$, the time is such that the maximum effective plastic strain is $\bar{\varepsilon} \sim 1.5$. Other simulation parameters are given in table 1.

\section{Ring expansion}

\subsection{Finite element model}

Three-dimensional numerical simulations of ring expansion have been performed using ABAQUS/Explicit (version 2017). The ring internal radius, external radius and height are $R_{\text {int }}=15.5 \mathrm{~mm}, R_{\text {ext }}=16.5 \mathrm{~mm}$, respectively, and $H_{e}=1 \mathrm{~mm}$. Initially, the ring occupies the domain $R_{\text {int }} \leq R \leq R_{\text {ext }}, 0 \leq \theta \leq 2 \pi, 0 \leq Z \leq H_{e}$ (R, $\theta$ and $Z$ being the cylindrical coordinates).

A constant radial velocity $V_{e}$ is applied on the internal surface of the specimen:

$$
V_{R}\left(R_{\text {int }}, \theta, Z, t\right)=V_{e}
$$

Other surfaces of the ring are traction free. The following initial velocity field is prescribed (Rodríguez-Martínez et al., 2013a):

$$
V_{R}(R, \theta, Z, 0)=V_{e}, \quad V_{\theta}(R, \theta, Z, 0)=0, \quad V_{Z}(R, \theta, Z, 0)=0
$$

The finite element mesh consists of 12550 hexahedral elements with trilinear interpolation functions, reduced integration and hourglass control (C3D8R in ABAQUS notation). The mesh has a radial symmetry and the dimensions of the elements are close to $0.2 \times 0.2 \times 0.2 \mathrm{~mm}^{3}$ (Fig. $10)$.

The development of necking instabilities is known to be very sensitive to imperfections (Hutchinson and Neale, 1977; Han and Tvergaard, 1995; Xavier et al., 2020; Marvi-Mashhadi and Rodríguez-Martínez, 2020). In the present simulations, a random material imperfection has been used to break the symmetry of the problem and favour necking localisation. The imperfection consists in a perturbation of the initial yield stress. A similar approach was used by Bishop and Strack (2011), Petit (2013) and El Maï (2014). In each element, the perturbed yield stress is defined as

$$
\tilde{\sigma}_{0}^{\delta}(i)=\sigma_{0}(1+\delta(\xi(i)-0.5))
$$

where $\sigma_{0}$ is the reference yield stress (see section 2 and table 1 ), $\delta$ is the imperfection magnitude, $i$ is the element number and $\xi(i)$ is random number of uniform probability density 
ranging between 0 and 1 . Note that for a given imperfection amplitude, it is possible to generate several initial yield stress distributions (varying the seed of the random number generator). The influence of stochastic uncertainties is outside the scope of the present paper. Therefore, all results presented in section 4.3 have been obtained with the same imperfection with $\delta=0.02$ (Fig. 10). However, we have carried out some simulations for other realisations of the imperfection and found almost the same necking characteristics (localisation time and strain, number of necks), see appendix.

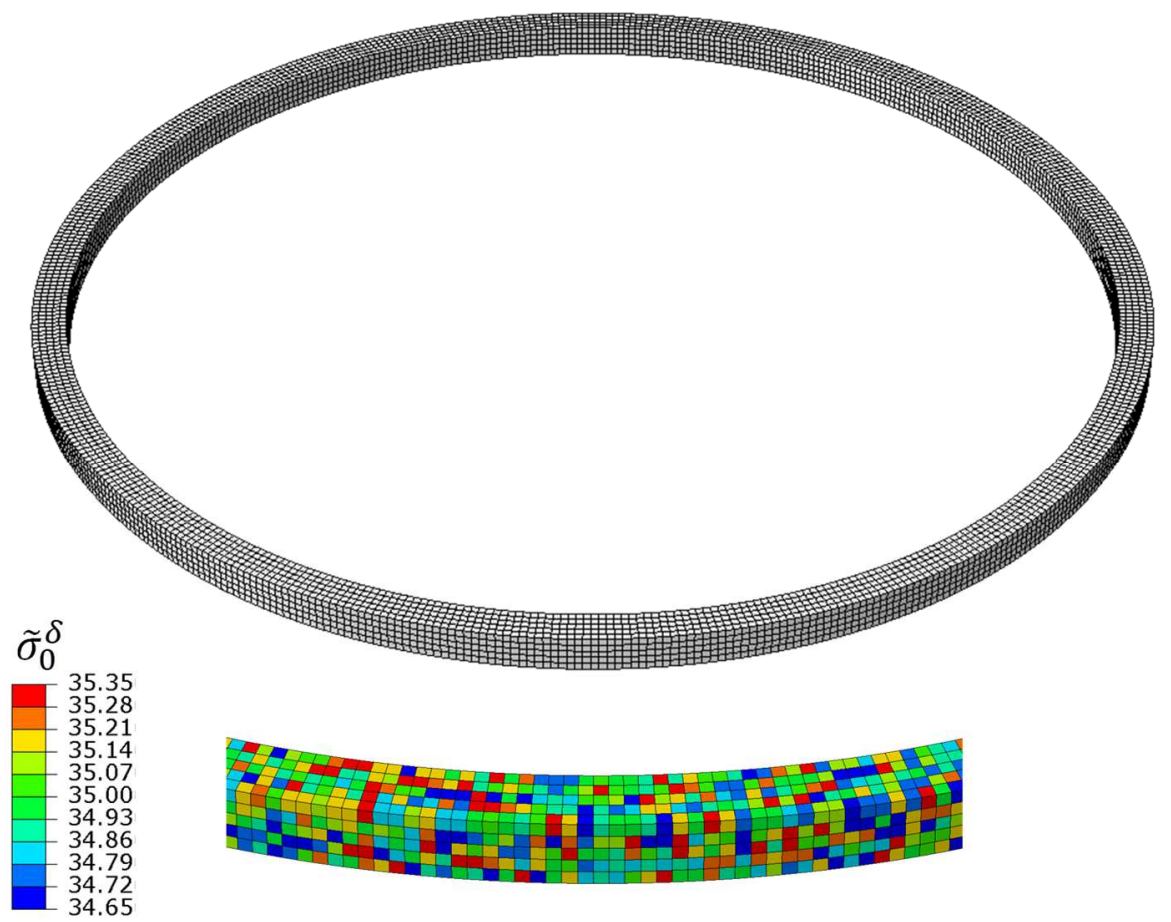

Fig. 10. Finite element mesh used in the ring expansion simulations. The bottom picture illustrates the distribution of initial yield stress (material imperfection).

\subsection{Linear stability analysis}

The stability analysis developed in this paper adapts the one-dimensional formulation of Zhou et al. (2006) to the constitutive model derived in section 2. As in Zhou et al. (2006), the elastic deformations are neglected, and the hydrostatic stresses that develop inside a necked section are modelled using Bridgman (1952) approximation. The ring expansion problem is approximated by a cylindrical bar with initial radius $r_{0}$, cross-section area $A_{0}=\pi r_{0}^{2}$ (the area $A_{0}$ is taken identical to that of the ring), and length $L_{0}=2 \pi \bar{R}$ ( $\bar{R}$ being the mean ring radius, 
$\bar{R}=\frac{R_{\text {int }}+R_{\text {ext }}}{2}$ ) subjected to axial velocity $V_{a}=2 \pi V_{e}\left(V_{e}\right.$ being the radial expansion velocity, see Eq. (13)) and spatially-uniform effective strain rate $\dot{\bar{\varepsilon}}$. The equations of the problem are (see Zhou et al. (2006) and Vadillo et al. (2012) for further details):

- Kinematic relations

$$
\begin{aligned}
& \frac{\partial v}{\partial X}=\dot{\bar{\varepsilon}} e^{\bar{\varepsilon}} \\
& \dot{\bar{\varepsilon}}=\frac{\partial \bar{\varepsilon}}{\partial t}
\end{aligned}
$$

- Momentum balance

$$
\frac{\partial\left(\sigma^{a v g} e^{-\bar{\varepsilon}}\right)}{\partial X}=\rho \frac{\partial v}{\partial t}
$$

- Average axial stress

$$
\sigma^{\text {avg }}=\bar{\sigma} B(\phi)
$$

- Bridgman (1952) correction factor

$$
\begin{aligned}
& B(\phi)=\left(1+\frac{1}{\phi}\right) \ln (1+\phi) \\
& \text { with } \quad \phi=\frac{1}{2} r \frac{\partial^{2} r}{\partial x^{2}}=\frac{A_{0} e^{-3 \bar{\varepsilon}}}{8 \pi}\left(3\left(\frac{\partial \bar{\varepsilon}}{\partial X}\right)^{2}-2\left(\frac{\partial^{2} \bar{\varepsilon}}{\partial X^{2}}\right)\right)
\end{aligned}
$$

where $X$ is the Lagrangian coordinate, $x$ is the Eulerian coordinate, $v$ is the axial velocity, and $r$ and $A$ are the current radius and cross-sectional area, respectively. The governing equations are completed with expressions (5) and (8), see section 2, which describe the evolution of the yield stress $\bar{\sigma}$ and the hardening variable $H$, and with the following initial and boundary conditions:

$$
v(X, 0)=\frac{V_{a}}{L_{0}} X \quad v(0, t)=0 \quad v\left(L_{0}, t\right)=V_{a}
$$

The homogeneous (or fundamental) solution of the problem is denoted by $\mathbb{S}_{1}=$ $\left(v_{1}, \bar{\varepsilon}_{1}, \dot{\bar{\varepsilon}}_{1}, \sigma_{1}^{a v g}, \bar{\sigma}_{1}, H_{1}, \phi_{1}\right)^{\mathrm{T}}$. At some time $t_{1}$, a perturbation $\delta \mathbb{S}(X, t)=\delta \mathbb{S}_{1} e^{i \xi X+\eta\left(t-t_{1}\right)}$ is inserted into the fundamental solution, so that the perturbed solution is $\mathbb{S}=\mathbb{S}_{1}+\delta \mathbb{S}$, where $\delta \mathbb{S}_{1}=\left(\delta v, \delta \bar{\varepsilon}, \delta \dot{\bar{\varepsilon}}, \delta \sigma^{a v g}, \delta \bar{\sigma}, \delta H, \delta \phi\right)^{T}$ is the perturbation amplitude, $\xi$ is the perturbation wavenumber (also called perturbation mode) and $\eta$ is the perturbation growth rate (also called instantaneous instability index). Assuming that $|\delta \mathbb{S}| \ll\left|\mathbb{S}_{1}\right|$, the following linearized equations are obtained:

- Kinematic relations

$$
\begin{aligned}
& \delta v+i \xi^{-1} e^{\bar{\varepsilon}_{1}}(\delta \dot{\bar{\varepsilon}}+\dot{\bar{\varepsilon}} \delta \bar{\varepsilon})=0 \\
& \eta \delta \bar{\varepsilon}-\delta \dot{\bar{\varepsilon}}=0
\end{aligned}
$$


- Momentum balance

$$
\rho \eta \delta v+i \xi \sigma_{1}^{a v g} e^{-\bar{\varepsilon}_{1}} \delta \bar{\varepsilon}-i \xi e^{-\bar{\varepsilon}_{1}} \delta \sigma^{a v g}=0
$$

- Average axial stress

$$
\delta \sigma^{a v g}-\delta \bar{\sigma}-\frac{1}{2} \bar{\sigma}_{1} \delta \phi=0
$$

- Bridgman (1952) correction factor

$$
\delta \phi-\frac{A_{0}}{4 \pi} \xi^{2} e^{-3 \bar{\varepsilon}_{1}} \delta \bar{\varepsilon}=0
$$

- Yield stress evolution

$$
\delta \bar{\sigma}-\left.\frac{\partial \bar{\sigma}}{\partial H}\right|_{t_{1}} \delta H-\left.\frac{\partial \bar{\sigma}}{\partial \dot{\bar{\varepsilon}}}\right|_{t_{1}} \delta \dot{\bar{\varepsilon}}=0
$$

- Hardening variable evolution

$$
\delta H-\left.\frac{\partial H}{\partial \bar{\varepsilon}}\right|_{t_{1}} \delta \bar{\varepsilon}=0
$$

A solution for $\delta \mathbb{S}_{1}$ is obtained when the determinant of the matrix of coefficients of equations (22)-(28) is zero. This condition leads to:

$$
B_{2} \hat{\eta}^{2}+B_{1} \hat{\eta}+B_{0}=0
$$

with $\quad B_{2}=1, \quad B_{1}=1+\hat{Q} \Lambda^{2} \widehat{L}^{2} \hat{\xi}^{2}, \quad B_{0}=\Lambda^{2} \widehat{L}^{2} \hat{\xi}^{2}\left(\hat{P} \hat{R}-\hat{\sigma}^{a v g}+\frac{\Lambda^{3} \hat{L}^{2} \widehat{\widehat{\sigma}}}{8}\right)$,

$$
\begin{aligned}
& \hat{\eta}=\frac{\eta}{\dot{\varepsilon}_{1}}, \quad \hat{\xi}=\xi \cdot r_{0}, \quad \Lambda=e^{-\bar{\varepsilon}_{1}}, \quad \hat{L}=\sqrt{\frac{\sigma_{0}}{\rho \dot{\bar{\varepsilon}}_{1}^{2} r_{0}^{2}}}, \quad \hat{P}=\left.\frac{\partial \bar{\sigma}}{\partial H}\right|_{t_{1}}, \quad \hat{Q}=\left.\frac{\dot{\varepsilon}_{1}}{\sigma_{0}} \frac{\partial \bar{\sigma}}{\partial \dot{\bar{\varepsilon}}}\right|_{t_{1}}, \\
& \hat{R}=\left.\frac{1}{\sigma_{0}} \frac{\partial H}{\partial \bar{\varepsilon}}\right|_{t_{1}}, \quad \hat{\sigma}^{a v g}=\left.\frac{\sigma^{a v g}}{\sigma_{0}}\right|_{t_{1}} \quad \text { and } \quad \hat{\sigma}=\left.\frac{\bar{\sigma}}{\sigma_{0}}\right|_{t_{1}}
\end{aligned}
$$

Equation (29) gives, for a given time $t_{1}$, the value of the dimensionless instantaneous instability index $\hat{\eta}$ as a function of the dimensionless perturbation mode $\hat{\xi}$, being the latter related to the dimensionless perturbation wavelength as $L_{0} / r_{0}=\frac{2 \pi}{\hat{\xi}}$. Fressengeas and Molinari (1994) proposed the cumulative instability index $I=\int_{0}^{t} \hat{\eta} \dot{\bar{\varepsilon}}_{1} \mathrm{~d} \tau$ as indicator of the stability of the solution. The wavelength of the mode having the largest cumulative instability index $I$ is the critical cumulative wavelength, and the maximum value of $I$ is the critical cumulative instability index $I^{c}$. The critical cumulative instability index will be used in section 4.3 to compare the results obtained with the linear stability analysis set out in this section, and the finite element model presented in section 4.1 . 


\subsection{Results}

Fig. 11 shows linear stability analysis results for the evolution of the critical cumulative instability index $I^{c}$ as a function of the effective strain $\bar{\varepsilon}$ for several values of the strain-rate history effect parameter $\alpha$ and two expansion velocities: $V_{e}=70 \mathrm{~m} / \mathrm{s}$ and $V_{e}=300 \mathrm{~m} / \mathrm{s}$ (corresponding to initial strain rates of $4375 \mathrm{~s}^{-1}$ and $18750 \mathrm{~s}^{-1}$, respectively). The critical cumulative index increases monotonically with the effective strain because thinning of the specimen promotes the formation of necking instabilities (Zaera et al., 2014). Moreover, increasing the applied velocity shifts downwards the $I^{c}-\bar{\varepsilon}$ curves due to the increase of inertia effects, slowing down the growth of the perturbation and stabilising material behaviour (Fressengeas and Molinari 1994, Rodríguez-Martínez et al. 2013a). Notice that the differences between the results obtained for the three different values of $\alpha$ are reduced as the expansion velocity increases. Notice also that the greater the strain-rate history effects (i.e. the greater the value of $\alpha$ ), the greater the gap between the results obtained for $70 \mathrm{~m} / \mathrm{s}$ and $300 \mathrm{~m} / \mathrm{s}$, and thus the effect of inertia slowing down the growth of the perturbation. In contrast, the instantaneous strain-rate sensitivity, while reducing the role of inertia, leads to greater material stabilisation. For instance, the $I^{c}-\bar{\varepsilon}$ curve for $\alpha=0$ and $V_{e}=70 \mathrm{~m} / \mathrm{s}$ is below the curve obtained for $\alpha=$ 0.5 and $V_{e}=300 \mathrm{~m} / \mathrm{s}$. These results make apparent that the relative contributions of inertia and rate dependence on material stabilisation depend on the specific nature of the strain-rate sensitivity, with the instantaneous strain-rate sensitivity playing a dominant role on the growth rate of the perturbation, and thus on the stability of the material and the formation of necking instabilities for the range of strain rates investigated in this work. 


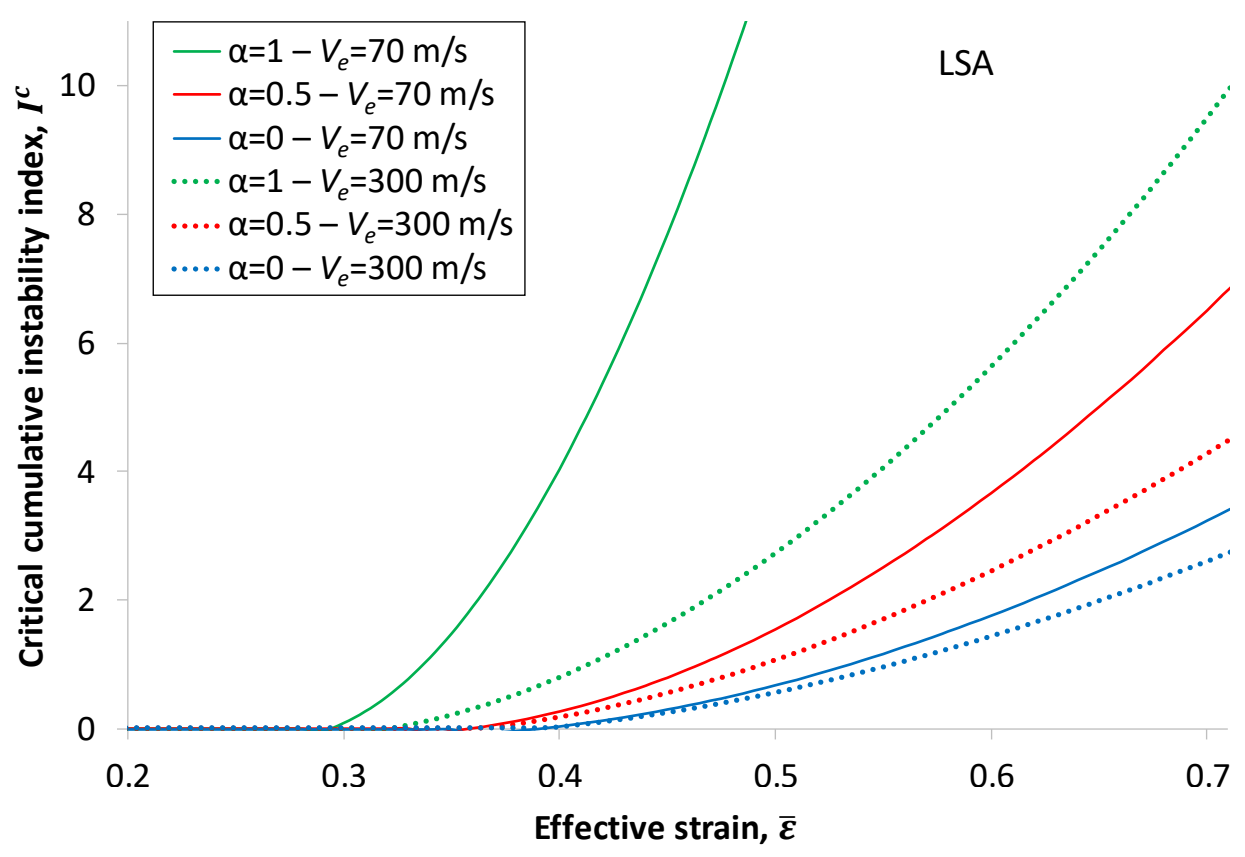

Fig. 11. Linear stability analysis results: evolution of the critical cumulative instability index $I^{c}$ with the effective strain $\bar{\varepsilon}$ for several values of the strain-rate history effect parameter $\alpha$ and two expansion velocities: $V_{e}=70 \mathrm{~m} / \mathrm{s}$ and $V_{e}=300 \mathrm{~m} / \mathrm{s}$ (corresponding to initial strain rates of $4375 \mathrm{~s}^{-1}$ and $18750 s^{-1}$, respectively). Other material parameters are given in table 1.

The greater stabilising role of instantaneous strain-rate sensitivity, in comparison with the workhardening strain-rate sensitivity, is further illustrated in Fig. 12, which shows finite element results for the normalised plastic strain rate $\hat{\bar{\varepsilon}}$ (ratio between the current and the background effective plastic strain rate) along the normalised circumferential coordinate, $\hat{P}=\theta / 2 \pi$, for $\alpha=0,0.5$ and 1 . The $\hat{\bar{\varepsilon}}-\hat{P}$ curves show a series of peaks and valleys. The peaks correspond to developing necks, while the valleys are the unloading portions of the ring surrounding the localising sections (the plastic strain rate is lower outside the necked sections). Recall from Fig. 5(b) that when a neck is formed the plastic strain rate increases drastically, leading to a heterogeneous distribution of plastic strains in the specimen. Therefore, the presence of numerous peaks in the $\hat{\bar{\varepsilon}}-\hat{P}$ curves indicates multiple plastic localisations. This phenomenon is commonly observed in dynamic ring expansion experiments (Altynova et al., 1996; Zhang and Ravi-Chandar, 2006, 2008). Results for $V_{e}=70 \mathrm{~m} / \mathrm{s}$ and $V_{e}=300 \mathrm{~m} / \mathrm{s}$ are shown in Figs. 12(a) and 12(b), respectively, with the corresponding loading times being $130 \mu \mathrm{s}$ and $53.7 \mu \mathrm{s}$ (the associated background strains are 0.45 and 0.7 ). The loading times have been chosen so that the maximum value of the normalised plastic strain rate for $\alpha=1$ is $\hat{\bar{\varepsilon}} \approx 1.65$. The 
heterogeneity in the distribution of plastic strain rates is significantly more pronounced in the case of $\alpha=1$, which shows that the material with pure work-hardening strain-rate sensitivity is prone to plastic localisation, noticeably more than the materials with (partial and full) instantaneous strain-rate sensitivity (which are more stable), in agreement with the predictions of the stability analysis (see Fig. 11). Notice also that the differences in the results obtained for $\alpha=0,0.5$ and 1 are reduced as the expansion velocity increases. Specifically, for $V_{e}=$ $300 \mathrm{~m} / \mathrm{s}$, the maximum value of $\hat{\bar{\varepsilon}}$ for $\alpha=0.5$ and $\alpha=0$ is 1.23 and 1.1 , respectively, while in the case of $V_{e}=70 \mathrm{~m} / \mathrm{s}$ the normalised plastic strain rate does not exceed 1.07 and 1.03 for these two values of $\alpha$. This is also consistent with the predictions of the stability analysis (see Fig. 11), which showed that, for a given effective plastic strain (i.e. for a given loading time), the differences in the critical cumulative instability index (i.e. the differences in the stability of the material) for $\alpha=0,0.5$ and 1 decrease with the expansion velocity. The reason of this phenomenon is that at very high expansion velocity, the neck retardation effect caused by inertia is enhanced and, therefore, the stabilising effect of the instantaneous strain-rate sensitivity becomes comparatively less important.

(a)

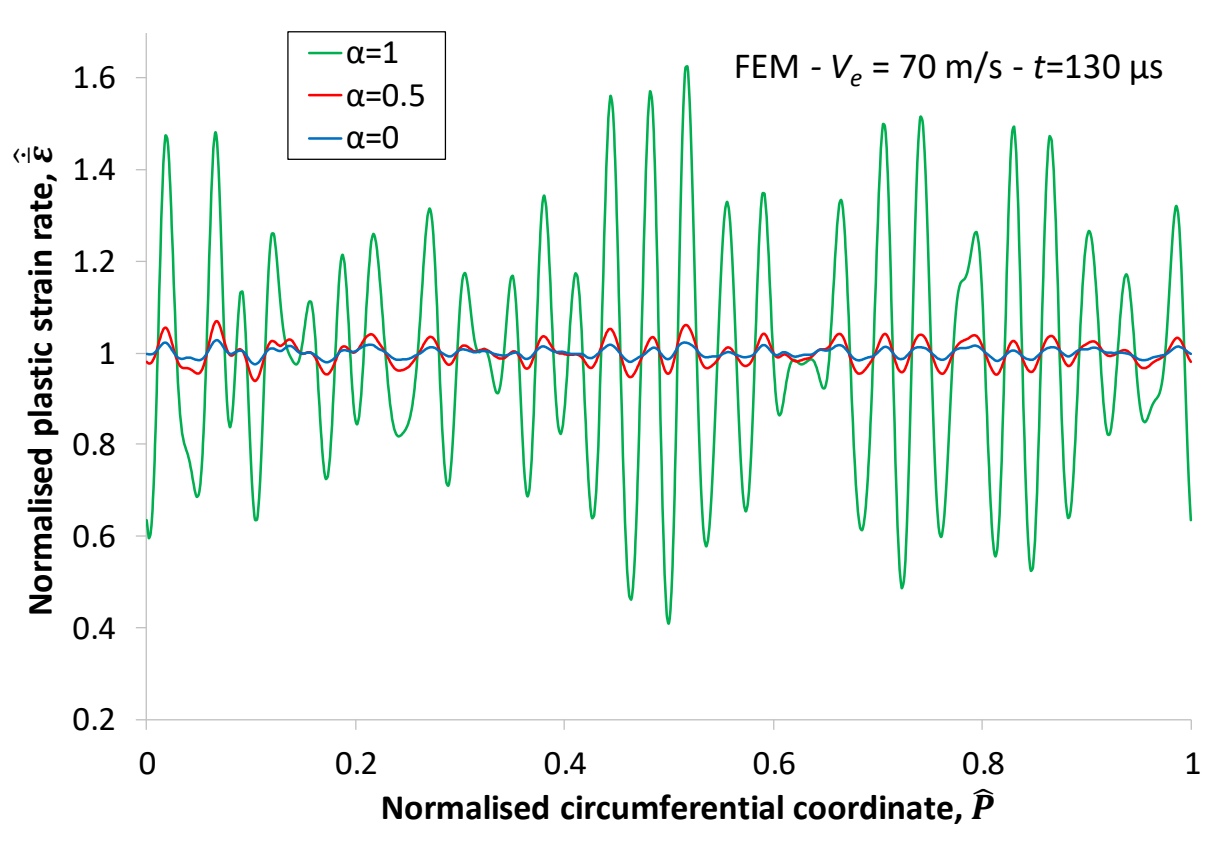

(b) 


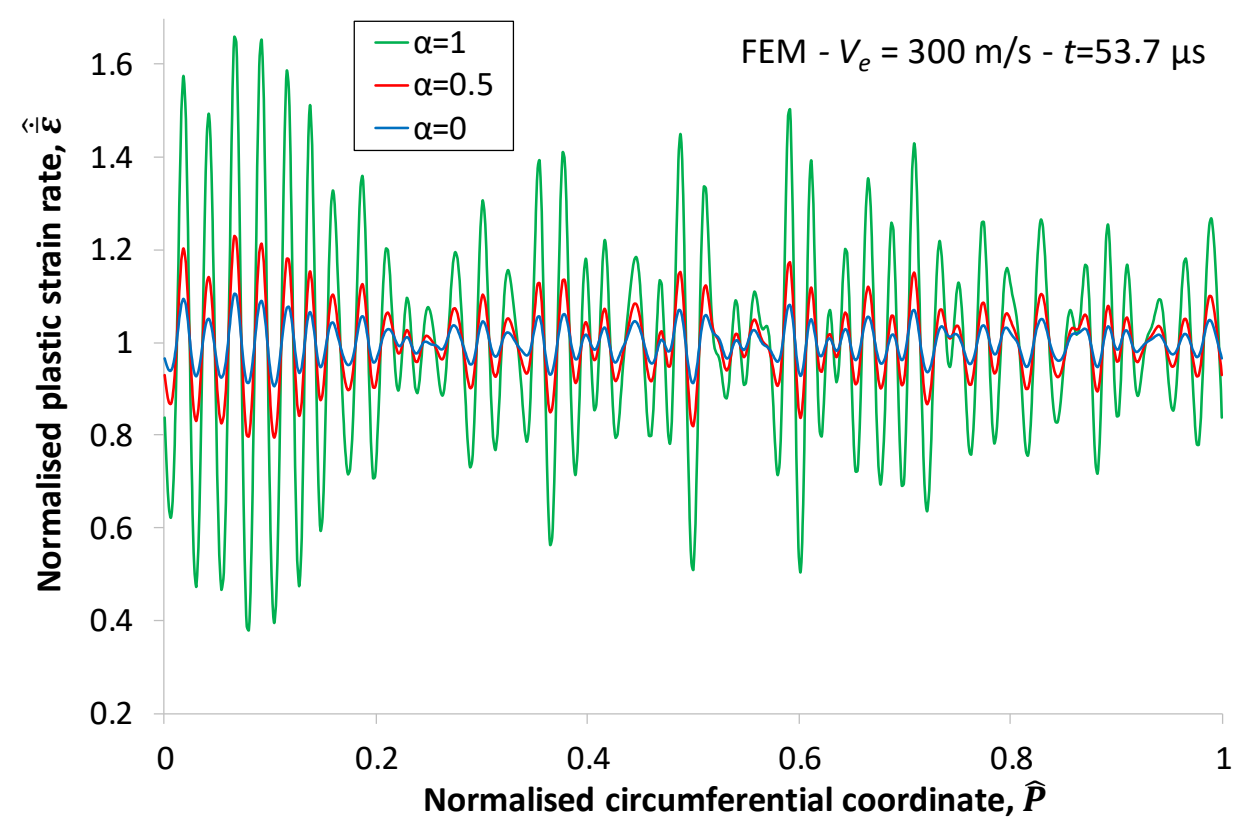

Fig. 12. Finite element results: normalised plastic strain rate $\hat{\bar{\varepsilon}}$ versus normalised circumferential coordinate $\widehat{P}$ for several values of $\alpha$, and different expansion velocities and loading times: (a) $V_{e}=$ $70 \mathrm{~m} / \mathrm{s}$ and $t=130 \mu \mathrm{s},(b) V_{e}=300 \mathrm{~m} / \mathrm{s}$ and $t=53.7 \mu \mathrm{s}$. The normalised plastic strain rate is defined as $\hat{\bar{\varepsilon}}=\dot{\bar{\varepsilon}} / \dot{\bar{\varepsilon}}_{b}$, where $\dot{\bar{\varepsilon}}_{b}$ is the background strain rate (average value of $\dot{\bar{\varepsilon}}$ along the ring circumference). The $\hat{\dot{\bar{\varepsilon}}}-\hat{P}$ curves show a series of peaks and valleys, so that the excursions of strain rate indicate multiple plastic localisations, and the valleys correspond to the unloading portions of the ring surrounding the localising sections. The loading times considered in Figs. 12-a and 12-b have been chosen in order to have nearly the same maximum value of the normalised plastic strain rate.

The corresponding values the background effective plastic strain are (a) 0.45 and (b) 0.7. Other material parameters are given in table 1.

The interplay between inertia and strain-rate history effects is further illustrated in Fig. 13, which shows the evolution of the necking strain $\bar{\varepsilon}^{\text {neck }}$ with the expansion velocity $V_{e}$, for $\alpha=$ $0,0.5$ and 1 . The finite element results are compared with the stability analysis predictions. In the numerical calculations, the necking strain corresponds to the minimum plastic strain in the inner perimeter of the ring when elastic unloading first occurs, i.e. when the plastic strain rate vanishes at any point of the internal surface of the specimen. The loading time corresponding to the necking strain is referred to as the necking time $t^{\text {neck }}$. Moreover, to determine the necking strain with the stability analysis, we have relied on the concept of effective instability introduced by Dudzinski and Molinari (1991), and later adopted by El Maï et al. (2014) and N'souglo et al. (2020, 2021), which states that localised necking occurs when the critical 
cumulative instability index reaches a reference value $I^{\text {neck }}$. In the present work, $I^{\text {neck }}$ is taken to be linearly dependent on the initial strain rate $^{3}$ (i.e. on the expansion velocity). Identification of the parameters $A$ and $B$ of the function $I^{\text {neck }}=A+B \cdot \dot{\bar{\varepsilon}}$ has been performed using the finite element results for the material with $\alpha=0$ (standard viscoplastic material with no strain-rate history effects). Specifically, for the extrema of the range of strain rates considered, $312.5 \mathrm{~s}^{-1}$ and $25000 \mathrm{~s}^{-1}$, we assume that $I^{\text {neck }}$ corresponds to the value of $I^{c}$ calculated with the fundamental solution of the problem corresponding to the necking strain. Thus, we have obtained the values $A=1.0025$ and $B=1.518 \cdot 10^{-4} \mathrm{~s}$, which are assumed to be valid also for the materials with $\alpha=0.5$ and $\alpha=1$.

The necking strain increases monotonically with the expansion velocity, due to the stabilizing effect of inertia, in agreement with the experimental observations and the numerical calculations reported by Altynova et al. (1996), Hu and Daehn (1996), and Guduru et al. (2002), among others. The linear stability analysis results are in qualitative agreement with the finite elements, with greater necking strains as the parameter $\alpha$ decreases (i.e. as the contribution of strain-rate history effects to material viscosity decreases). It becomes apparent that the analytical model captures the underlying mechanisms which determine the respective influence of instantaneous strain-rate sensitivity and work-hardening strain-rate sensitivity on necking localisation. On the other hand, the quantitative differences between numerical calculations and analytical predictions increase with $\alpha$. In this regard, recall that $I^{\text {neck }}$ was calibrated using the numerical results obtained for $\alpha=0$, which explains the perfect matching between finite element calculations and stability analysis obtained for this material for the extrema of the range of expansion velocities considered, and consequently the small differences observed for intermediates values of $V_{e}$. For $\alpha=0.5$ and $\alpha=1$, the stability analysis predictions underestimate the finite element results for most velocities tested, with a gap that reaches $18 \%$ in the case of the material showing pure work-hardening strain-rate sensitivity $(\alpha=1)$. Moreover, notice that for $\alpha=1$ and the lower expansion velocity $V_{e}=5 \mathrm{~m} / \mathrm{s}$, the necking strain is only slightly greater than the instability strain associated to the Considère criterion. This shows that, as in the case of the bar under impact tension, the work-hardening strain-rate sensitivity does not yield neck retardation effect. In contrast, for $\alpha=0$ and 0.5 , and the same expansion velocity of $5 \mathrm{~m} / \mathrm{s}$ (for which the contribution of inertia is negligible), the necking strain is significantly higher, $\sim 0.45$ and $\sim 0.55$, respectively, showing the material stabilisation

\footnotetext{
${ }^{3}$ Dudzinski and Molinari (1991), El Maï et al. (2014) and N'souglo et al. $(2020,2021)$ assumed that $I^{\text {neck }}$ is constant.
} 
induced by instantaneous strain-rate sensitivity at low strain rates. Even if there is not a perfect quantitative agreement between the finite element computations and the linear stability analysis, the important observation is that both approaches predict a significant influence of strain-rate history effects on ductility. When the relative contribution of the work-hardening strain-rate sensitivity increases, the necking strain decreases. Contrary to the case of the bar under impact tension investigated in section 3 , there is no critical velocity for the ring expansion problem (Hu and Daehn, 1996). Therefore, the influence of strain-rate history effects remains significant in the whole range of expansion velocities considered. However, the differences between the necking strains corresponding to the three values of $\alpha$ tend to decrease for very high expansion velocities, as the stabilising effect of inertia becomes more important.

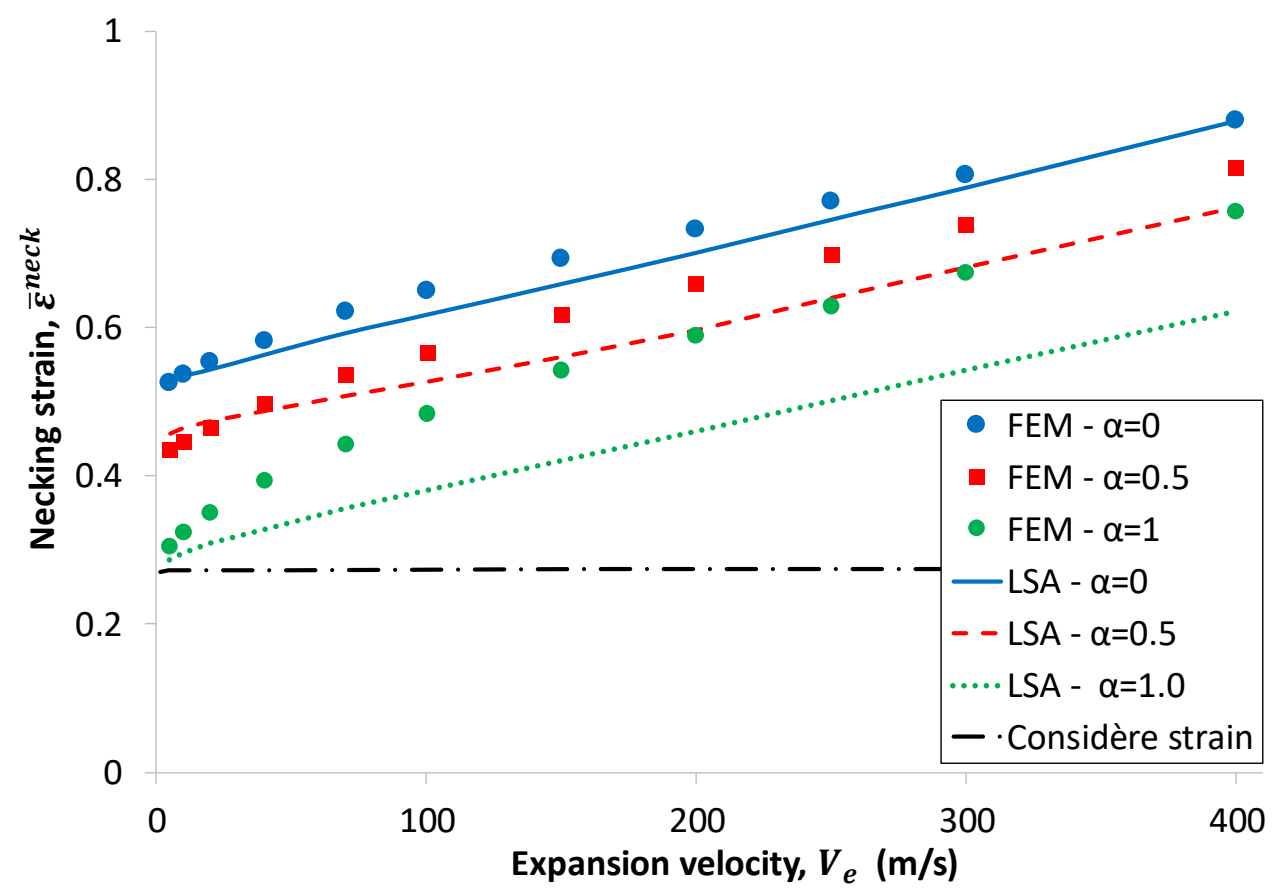

Fig. 13. Evolution of the necking strain $\bar{\varepsilon}^{\text {neck }}$ as a function of the expansion velocity $V_{e}$ for several values of the strain-rate history effect parameter $\alpha$. Comparison between finite element results and linear stability analysis predictions. The instability strain associated to the Considère criterion is also shown. Other material parameters are given in table 1. 


\section{Concluding remarks}

We have investigated the development of necking instabilities in materials exhibiting strainrate history effects. Two different problems have been considered: a bar under impact tension and a ring subjected to rapid radial expansion. For these two problems, finite element computations using a constitutive model that takes strain-rate history effects into account have been carried out. Moreover, for the ring expansion problem, the one-dimensional linear stability analysis introduced by (Zhou et al., 2006) has been extended to consider strain-rate history effects. The results presented in this paper show that strain-rate history effects may have a significant influence on neck formation. For materials presenting strain-rate history effects, two different strain-rate sensitivities are operative: the instantaneous strain-rate sensitivity and the strain-rate sensitivity of work hardening. We have analysed the influence of these two kinds of strain-rate sensitivities on neck formation. The main finding is that only the instantaneous strain-rate sensitivity leads to neck retardation effect, when the neck formation is related to a plastic flow instability.

In the case of the bar under impact tension, we have observed that the influence of strain-rate history effects vanishes when the applied velocity reaches the critical impact velocity (CIV). For supercritical velocities, necking is no longer caused by a plastic flow instability, but is due to wave trapping. The strain-rate history effects do not significantly influence the propagation of plastic waves so that necking formation under supercritical loading conditions is largely insensitive to strain-rate history effects. Note that no critical velocity exists for the expanding ring configuration and therefore strain-rate history effects on necking remain significant even for very high expansion velocities.

An implication of the results presented in this paper is that the experimental characterisation of the constitutive behaviour of materials should not only rely on tests at constant strain rate if the analysis of plastic flow instabilities is of concern. Tests with strain-rate jumps should also be performed to determine if the material exhibits strain-rate history effects. If this is the case, specific constitutive models taking these effects into account should be employed to analyse the development of necking instabilities. 


\section{Acknowledgements}

J.A.R.-M acknowledges that the research leading to these results has received funding from the European Research Council (ERC) under the European Union's Horizon 2020 research and innovation programme. Project PURPOSE, grant agreement 758056.

\section{Appendix: stochastic variations in the finite element ring expansion simulations}

The ring expansion simulations described in section 4.1 involve a random material imperfection. For a given defect amplitude, it is possible to create several realisations of the imperfection (i.e. several distributions of initial yield stress). Table A.1 provides numerical results corresponding to 5 different realisations of an imperfection of amplitude $\delta=0.02$ (see Eq. (15)) for an expansion velocity $V_{e}=70 \mathrm{~m} / \mathrm{s}$ and several values of the strain-rate history effect parameter $\alpha$. The necking time (time at which elastic unloading first occurs), the necking strain and the number of necks (as defined in section 4.3) are presented. For these three quantities, the five realisations yield very close results. The relative standard deviation is lower than $2 \%$ for the necking time and the necking strain, and about $5 \%$ for the number of necks.

\begin{tabular}{|c|c|c|c|c|c|c|c|c|c|}
\hline \multirow{2}{*}{$\begin{array}{c}\text { Realisation } \\
\#\end{array}$} & \multicolumn{3}{|c|}{ Necking time (ms) } & \multicolumn{3}{c|}{ Necking strain } & \multicolumn{3}{c|}{ Number of necks } \\
\cline { 2 - 11 } & $\alpha=0$ & $\alpha=0.5$ & $\alpha=1$ & $\alpha=0$ & $\alpha=0.5$ & $\alpha=1$ & $\alpha=0$ & $\alpha=0.5$ & $\alpha=1$ \\
\hline 1 & 0.234 & 0.191 & 0.135 & 0.622 & 0.537 & 0.443 & 23 & 25 & 26 \\
\hline 2 & 0.232 & 0.188 & 0.134 & 0.612 & 0.53 & 0.44 & 26 & 28 & 29 \\
\hline 3 & 0.224 & 0.183 & 0.129 & 0.587 & 0.508 & 0.424 & 26 & 26 & 27 \\
\hline 4 & 0.234 & 0.189 & 0.134 & 0.614 & 0.528 & 0.439 & 28 & 28 & 29 \\
\hline 5 & 0.229 & 0.186 & 0.133 & 0.602 & 0.527 & 0.437 & 25 & 25 & 29 \\
\hline Mean value & 0.231 & 0.188 & 0.133 & 0.609 & 0.526 & 0.437 & 25.6 & 26.4 & 28 \\
\hline $\begin{array}{c}\text { Relative } \\
\text { standard } \\
\text { deviation } \\
(\%)\end{array}$ & 1.58 & 1.52 & 1.42 & 1.93 & 1.825 & 1.47 & 6.35 & 5.14 & 4.52 \\
\hline
\end{tabular}

Table A.1. Necking time, necking strain and number of necks obtained with ring expansion simulations for several realisations of a random imperfection of amplitude $\delta=0.02$ (see Eq. (15)) and several 
values of the strain-rate history effect parameter $\alpha$. The expansion velocity is $V_{e}=70 \mathrm{~m} / \mathrm{s}$ (corresponding to an initial strain rate of $4375 \mathrm{~s}^{-1}$ ).

\section{References}

Altynova M., Hu X., Daehn G.S., 1996. Increased ductility in high velocity electromagnetic ring expansion. Metallurgical and Materials Transactions A, 27(7), 1837-1844.

Audoly B., Hutchinson J.W., 2019. One-dimensional modeling of necking in rate-dependent materials. Journal of the Mechanics and Physics of Solids, 123, 149-171.

Bishop J.E., Strack O.E., 2011. A statistical method for verifying mesh convergence in Monte Carlo simulations with application to fragmentation. International Journal for Numerical Methods in Engineering, 88(3), 279-306.

Bodner S.R., Rubin M.B., 1994. Modeling of hardening at very high strain rates. Journal of Applied Physics, 76(5), 2742-2747.

Bridgman P.W., 1952. Studies in large plastic flow and fracture, with special emphasis on the effects of hydrostatic pressure. McGRAW-HILL, New-York.

Chiem C.Y., Duffy J., 1983. Strain rate history effects and observations of dislocation substructure in aluminum single crystals following dynamic deformation. Materials Science and Engineering, 57(2), 233-247.

Chung K., Wagoner R.H., 1988. Effects of work-hardening and rate sensitivity on the sheet tensile test. Metallurgical Transactions A, 19(2), 293-300.

Clark, D.S., Duwez, P.E., 1948. Discussion of the forces acting in tension impact test of materials. Journal of Applied Mechanics, 15, A243.

Clark D.S., Wood D.S., 1950. The influence of specimen dimension and shape on the results in tension impact testing. Proceedings - American Society for Testing and Materials, 50, 577-585.

dos Santos T., Rosa P.A., Maghous S., Rossi R., 2016. A simplified approach to high strain rate effects in cold deformation of polycrystalline FCC metals: Constitutive formulation and model calibration. International Journal of Plasticity, 82, 76-96.

El Maï S., 2014. Étude du développement des instabilités dans un anneau en expansion dynamique. Doctoral dissertation, Université de Lorraine. https://hal.univ-lorraine.fr/tel-01750799 
El Maï S., Mercier S., Petit J., Molinari A., 2014. An extension of the linear stability analysis for the prediction of multiple necking during dynamic extension of round bar. International Journal of Solids and Structures, 51, 3491-3507.

Estrin Y., Mecking H., 1984. A unified phenomenological description of work hardening and creep based on one-parameter models. Acta Metallurgica, 32(1), 57-70.

Follansbee P.S., Kocks U.F., 1988. A constitutive description of the deformation of copper based on the use of the mechanical threshold stress as an internal state variable. Acta Metallurgica, 36(1), 81-93.

French I.E., Weinrich P.F., 1975. The influence of hydrostatic pressure on the tensile deformation and fracture of copper. Metallurgical Transactions A, 6(4), 785.

Fressengeas C., Molinari A., 1985. Inertia and thermal effects on the localization of plastic flow. Acta Metallurgica, 33(3), 387-396.

Fressengeas C., Molinari A., 1994. Fragmentation of rapidly stretching sheets. European Journal of Mechanics, A/Solids, 13(2), 251-268.

Ghosh A.K., 1977a. Tensile instability and necking in materials with strain hardening and strain-rate hardening. Acta Metallurgica, 25(12), 1413-1424.

Ghosh A.K., 1977b. The influence of strain hardening and strain-rate sensitivity on sheet metal forming. Journal of Engineering Materials and Technology, 99(3), 264-274.

Gould P.J., Goldthorpe B.D., 2000. A path-dependent constitutive model for gilding copper. Le Journal de Physique IV, 10(PR9), 39-44.

Grady D.E., Benson D.A., 1983. Fragmentation of metal rings by electromagnetic loading. Experimental Mechanics, 23(4), 393-400.

Grady D.E., Olsen M.L., 2003. A statistics and energy based theory of dynamic fragmentation. International Journal of Impact Engineering, 29, 293-306.

Guduru P.R., Freund L.B., 2002. The dynamics of multiple neck formation and fragmentation in high rate extension of ductile materials. International Journal of Solids and Structures, 39, 5615-5632.

Han J.B., Tvergaard V., 1995. Effect of inertia on the necking behaviour of ring specimens under rapid radial expansion. European Journal of Mechanics, A/Solids, 14(2), 287-307.

Hu X., Daehn G.S., 1996. Effect of velocity on flow localization in tension. Acta Materialia, 44(3), 1021-1033.

Hutchinson J.W., Neale K.W., 1977. Influence of strain-rate sensitivity on necking under uniaxial tension. Acta Metallurgica, 25(8), 839-846. 
Jacques N., 2020. An analytical model for necking strains in stretched plates under dynamic biaxial loading. International Journal of Solids and Structures, 200-201, 198-212.

Jeanson A.C., Bay F., Jacques N., Avrillaud G., Arrigoni M., Mazars G., 2016. A coupled experimental/numerical approach for the characterization of material behaviour at high strain-rate using electromagnetic tube expansion testing. International Journal of Impact Engineering, 98, 75-87.

Klepaczko J., 1975. Thermally activated flow and strain rate history effects for some polycrystalline FCC metals. Materials Science and Engineering, 18(1), 121-135.

Klepaczko J.R., 2005. Review on critical impact velocities in tension and shear. International Journal of Impact Engineering, 32(1-4), 188-209.

Klepaczko J.R., Chiem C.Y., 1986. On rate sensitivity of FCC metals, instantaneous rate sensitivity and rate sensitivity of strain hardening. Journal of the Mechanics and Physics of Solids, 34(1), 29-54.

Lea L.J., 2018. Structural evolution in the dynamic plasticity of FCC metals. Doctoral dissertation, University of Cambridge. https://doi.org/10.17863/CAM.20971

Mann H.C., 1936. High-velocity tension-impact tests. Proceedings - American Society for Testing and Materials, 36-85.

Marciniak Z., Kuczyński K., Pokora T., 1973. Influence of the plastic properties of a material on the forming limit diagram for sheet metal in tension. International Journal of Mechanical Sciences, 15(10), 789-800.

Marvi-Mashhadi M., Rodríguez-Martínez J.A., 2020. Multiple necking patterns in elasto-plastic rings subjected to rapid radial expansion: The effect of random distributions of geometric imperfections. International Journal of Impact Engineering, 144, 103661.

Mercier S., Molinari A., 2003. Predictions of bifurcation and instabilities during dynamic extension. International Journal of Solids and Structures, 40(8), 1995-2016.

Mirone G., Barbagallo R., Giudice F., 2019. Locking of the strain rate effect in Hopkinson bar testing of a mild steel. International Journal of Impact Engineering, 130, 97-112.

Mirone G., Barbagallo R., Giudice F., Di Bella S., 2020. Analysis and modelling of tensile and torsional behaviour at different strain rates of Ti6Al4V alloy additive manufactured by electron beam melting (EBM). Materials Science and Engineering: A, 793, 139916.

Molinari A., Ravichandran G., 2005. Constitutive modeling of high-strain-rate deformation in metals based on the evolution of an effective microstructural length. Mechanics of Materials, 37(7), 737-752.

N'souglo K.E., Jacques N, Rodríguez-Martínez J.A., 2021. A three-pronged approach to predict the effect of plastic orthotropy on the formability of thin sheets subjected to dynamic biaxial stretching. Journal of the Mechanics and Physics of Solids, 146, 104189. 
N'souglo K.E., Rodríguez-Martínez J.A., Cazacu O., 2020. The effect of tension-compression asymmetry on the formation of dynamic necking instabilities under plane strain stretching. International Journal of Plasticity, 128, 102656.

N'souglo K.E., Srivastava A., Osovski S., Rodríguez-Martínez J.A., 2018. Random distributions of material defects trigger regular necking patterns at high strain rates. Proceedings of the Royal Society A: Mathematical, Physical and Engineering Sciences, 474, 20170575.

Needleman A., 2018. Effect of size on necking of dynamically loaded notched bars. Mechanics of Materials, 116, 180-188.

Nemes J.A., Eftis, J., 1993. Constitutive modeling of the dynamic fracture of smooth tensile bars. International Journal of Plasticity, 9(2), 243-270.

Nicholas T., 1971. Strain-rate and strain-rate-history effects in several metals in torsion. Experimental Mechanics, 11(5), 370-374.

Niordson F.I., 1965. A unit for testing materials at high strain rates. Experimental Mechanics, 5(1), 2932.

Osovski S., Rittel D., Rodríguez-Martínez J. A., Zaera R., 2013. Dynamic tensile necking: influence of specimen geometry and boundary conditions. Mechanics of Materials, 62, 1-13.

Petit J., 2013. An approach to generate random localizations in Lagrangian numerical simulations. Materials under Extreme Loadings: Application to Penetration and Impact, 311-332. https://doi.org/10.1002/9781118622612.ch14

Rittel D., Rotbaum Y., Rodríguez-Martínez J.A., Sory D., Zaera R., 2014. Dynamic necking of notched tensile bars: an experimental study. Experimental Mechanics, 54(6), 1099-1109.

Rodríguez-Martínez J.A., Rittel D., Zaera R., Osovski S., 2013b. Finite element analysis of AISI 304 steel sheets subjected to dynamic tension: the effects of martensitic transformation and plastic strain development on flow localization. International Journal of Impact Engineering, 54, 206-216.

Rodríguez-Martínez J.A., Vadillo G., Fernández-Sáez J., Molinari A., 2013a. Identification of the critical wavelength responsible for the fragmentation of ductile rings expanding at very high strain rates. Journal of the Mechanics and Physics of Solids, 61(6), 1357-1376.

Rotbaum Y., Osovski S., Rittel D., 2015. Why does necking ignore notches in dynamic tension?. Journal of the Mechanics and Physics of Solids, 78, 173-185.

Rusinek A., Zaera R., Klepaczko J.R., Cheriguene R., 2005. Analysis of inertia and scale effects on dynamic neck formation during tension of sheet steel. Acta Materialia, 53(20), 5387-5400.

Senseny P.E., Duffy J., Hawley R.H., 1978. Experiments on strain rate history and temperature effects during the plastic deformation of close-packed metals. Journal of Applied Mechanics, 45(1), 60-66. 
Simo J.C., Hughes T.J., 1998. Computational inelasticity. Springer-Verlag New-York, Inc.

Tong W., Clifton R.J., Huang S., 1992. Pressure-shear impact investigation of strain rate history effects in oxygen-free high-conductivity copper. Journal of the Mechanics and Physics of Solids, 40(6), 12511294.

Vadillo G., Rodríguez-Martínez J.A., Fernández-Sáez J., 2012. On the interplay between strain rate and strain rate sensitivity on flow localization in the dynamic expansion of ductile rings. International Journal of Solids and Structures, 49(3-4), 481-491.

Vaz-Romero A., Rodríguez-Martínez J.A., 2019. A one-dimensional model to describe flow localization in viscoplastic slender bars subjected to super critical impact velocities. Mechanics of Time-Dependent Materials, 23(1), 75-95.

Vaz-Romero A., Rodríguez-Martínez J.A., Arias A., 2015. The deterministic nature of the fracture location in the dynamic tensile testing of steel sheets. International Journal of Impact Engineering, 86, 318-335.

Vaz-Romero A., Rodríguez-Martínez J.A., Mercier S., Molinari A., 2017. Multiple necking pattern in nonlinear elastic bars subjected to dynamic stretching: The role of defects and inertia. International Journal of Solids and Structures, 125, 232- 243.

von Karman T., Duwez P., 1950. The propagation of plastic deformation in solids. Journal of Applied Physics, 21(10), 987-994.

Wagoner R.H., Wang N.M., 1983. Operant strain-rate sensitivity during tensile necking. Metallurgical Transactions A, 14(11), 2395-2406.

Walley S.M., Church P.D., Townsley R., Field, J. E., 2000. Validation of a path-dependent constitutive model for FCC and BCC metals using "symmetric" Taylor impact. Journal de Physique IV, 10(PR9), Pr9-69.

Wood W.W., 1967. Experimental mechanics at velocity extremes - very high strain rates. Experimental Mechanics, 7(10), 441-446.

Woodford, D.A., 1969. Strain-rate sensitivity as a measure of ductility (strain-rate sensitivity measurements to determine ductility of materials, noting relation to elongation). ASM. Trans. Q. 62, 291-293.

Xavier M., Czarnota C., Jouve D., Mercier S., Dequiedt J.L., Molinari A., 2020. Extension of linear stability analysis for the dynamic stretching of plates: Spatio-temporal evolution of the perturbation. European Journal of Mechanics, A/Solids, 79, 103860.

Xue Z., Vaziri A., Hutchinson J.W., 2008. Material aspects of dynamic neck retardation. Journal of the Mechanics and Physics of Solids, 56(1), 93-113. 
Zaera R., Rodríguez-Martínez J.A., Vadillo G., Fernández-Sáez J., 2014. Dynamic necking in materials with strain induced martensitic transformation. Journal of the Mechanics and Physics of Solids, 64(1), 316-337.

Zerilli F.J., Armstrong R.W., 1987. Dislocation-mechanics-based constitutive relations for material dynamics calculations. Journal of Applied Physics, 61(5), 1816-1825.

Zhang H., Ravi-Chandar K., 2006. On the dynamics of necking and fragmentation-I. Real-time and post-mortem observations in Al 6061-O. International Journal of Fracture, 142(3-4), 183-217.

Zhang H., Ravi-Chandar K., 2008. On the dynamics of necking and fragmentation-II. Effect of material properties, geometrical constraints and absolute size. International Journal of Fracture, 150(12), 3-36.

Zhang H., Ravi-Chandar K., 2010. On the dynamics of localization and fragmentation-IV. Expansion of Al 6061-O tubes. International Journal of Fracture, 163(1-2), 41-65.

Zhou F., Molinari J.F., Ramesh K.T., 2006. An elastic-visco-plastic analysis of ductile expanding ring. International Journal of Impact Engineering, 33(1-12), 880-891. 